\title{
Nele Dittrich
}

\section{Wenn die konkret dreimal klingelt - Die Horst Gärtner-Affäre}

Die 68er-Bewegung in der Landeshauptstadt Kiel war im Vergleich zu den übrigen Protestzentren innerhalb der Bundesrepublik und West-Berlins ein wohl eher kleinerer Schauplatz dieses transnationalen Phänomens. Dennoch lassen sich auch hier die spezifischen Merkmale der westdeutschen Studentenbewegung von 1968 feststellen. Die Christian-Albrechts-Universität zu Kiel (CAU) wurde zur Bühne verschiedener Aktionsformen wie Sit-ins, Muff-outs, Teach-ins, Go-ins - ausgeführt von ihren Studierenden.

Wie die Festschrift zum 350-jährigen Jubiläum der Christiana Albertina aus dem Jahr 2015 zeigt, sind bereits einige Beiträge zur Universitätsgeschichte mit Hinblick auf die Studentenbewegung geleistet worden. $\underline{2}$ Der Beitrag zur Horst Gärtner-Affäre knüpft an die bisherige Forschung an und leistet somit einen weiteren Beitrag zur Aufarbeitung der 68er-Studentenbewegung in Kiel, deren Methoden gleichzeitig als ein generelles Beispiel der gesamten Bewegung in West-Deutschland und West-Berlin angesehen werden können.

Die sogenannte Horst Gärtner-Affäre verdeutlicht sowohl die diskreditierenden Methoden der Kieler und Hamburger Protestbewegungen gegen Professoren als auch die allgemeinen Möglichkeiten, die Professor Horst Gärtner und dem Kultusministerium zur Verfügung standen, um sich gegen diese zu wehren. Daher werden nicht nur die Protestierenden, bestehend aus Studierenden und Journalisten, sondern auch Professor Horst Gärtner und die Universität gleichermaßen beleuchtet. Es wird nach der Vorgehensweise und Motivation der einzelnen Akteure gefragt und ihr Verhalten im Hinblick auf die bundesdeutsche Entwicklung der 68er-Bewegung kontextualisiert. Aus welcher Motivation heraus kam es seitens der Journalisten und Studierenden zu solchen Beschuldigungen gegen Professor Gärtner, die sowohl dessen Forschungen wie auch die des gesamten Hygiene-Institutes diskreditierten? Wie konnte ein Hochschullehrer beziehungsweise die Institution Universität dagegen vorgehen? Als Akteure oder Akteursgruppen werden die konkret-Journalisten, die drei angeklagten Kieler Studenten, Horst Gärtner und andere Professoren, der Senat der Universität Kiel sowie das Kultusministerium näher betrachtet. $\underline{\underline{3}}$

Ende September 1969 ging ein fingierter Telefonanruf der Hamburger Zeitschrift konkret beim Kieler Ordinarius für Virologie und Leiter des Hygienischen Instituts Horst Gärtner ein. Am 2. Oktober erschien daraufhin in der Hamburger Zeitschrift konkret die Reportage „Giftgas für die Bundeswehr“ mit dem zusätzlichen Beitrag „Umfrage bei Giftmischern“, der ein Interview mit Gärtner und weiteren Kollegen 


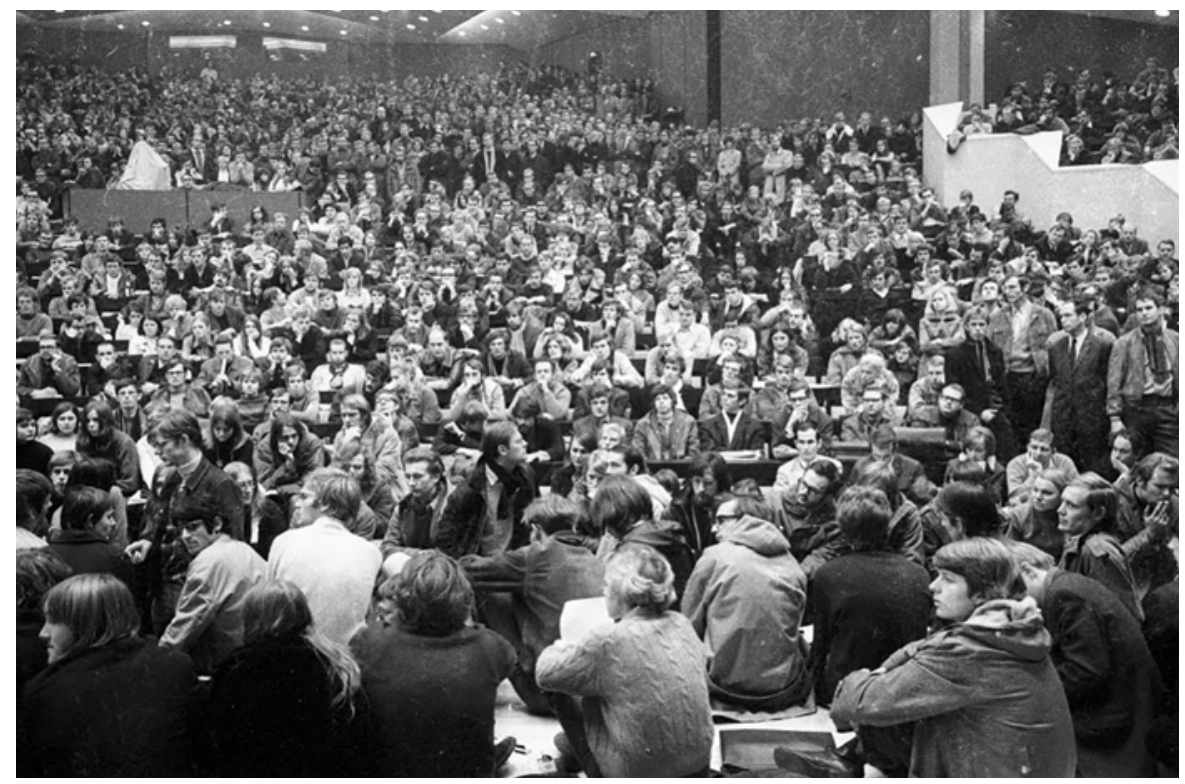

Abb. 1: Studentisches Teach-in im überfüllten Audimax der CAU (27.11.1969).

(C) 1969 Friedrich Magnussen, veröffentlicht unter der Lizenz CC-BY-SA 3.0 DE,

Stadtarchiv Kiel, Sign. 22.036.

wiedergab. Basierend auf den fingierten Telefonanrufen wurde ihnen durch eine verfälschte Wiedergabe des Interviews unterstellt, an der Entwicklung von B- und C-Waffen mitzuarbeiten oder zumindest ein Interesse an einem solchen Forschungsvorhaben zu zeigen. Bereits am 12. Oktober folgte in dem studentischen Kieler Informationsblatt AStA-info der Artikel „Kriegsforschung in Kiel oder Gärtner muß Gärtner werden“, dem die konkret-Reportage mit den Vorwürfen gegen Gärtner als einzige Quelle zugrunde lag. Auf einer Kieler Senatssitzung am 14. Oktober wurde die Klärung der Vorwürfe gegen Professor Gärtner als erster Tagesordnungspunkt behandelt. Die anwesenden studentischen Senatsvertreter Stabel und der Vorsitzende des Allgemeinen Studenten-Ausschusses (AStA) der CAU Dietmar Schlinke verlangten die Amtsenthebung des Ordinarius und die Offenlegung aller Forschungsprojekte sowie deren Finanzierungen durch das Hygiene-Institut. ${ }^{4}$ Des Weiteren wurde eine Untersuchungskommission zusammengestellt, die besagtes Institut auf etwaige Kriegsforschung untersuchen sollte. Bereits am 16. Oktober ließ der Kultusminister in einer Pressemitteilung verlautbaren, dass geprüft werde, ob „die Darstellungen der Zeitschrift und des studentischen Informationsblattes strafbar" seien, und dass er als Dienstvorgesetzter Professor Gärtners „die notwendigen Strafanträge“ gestellt habe 


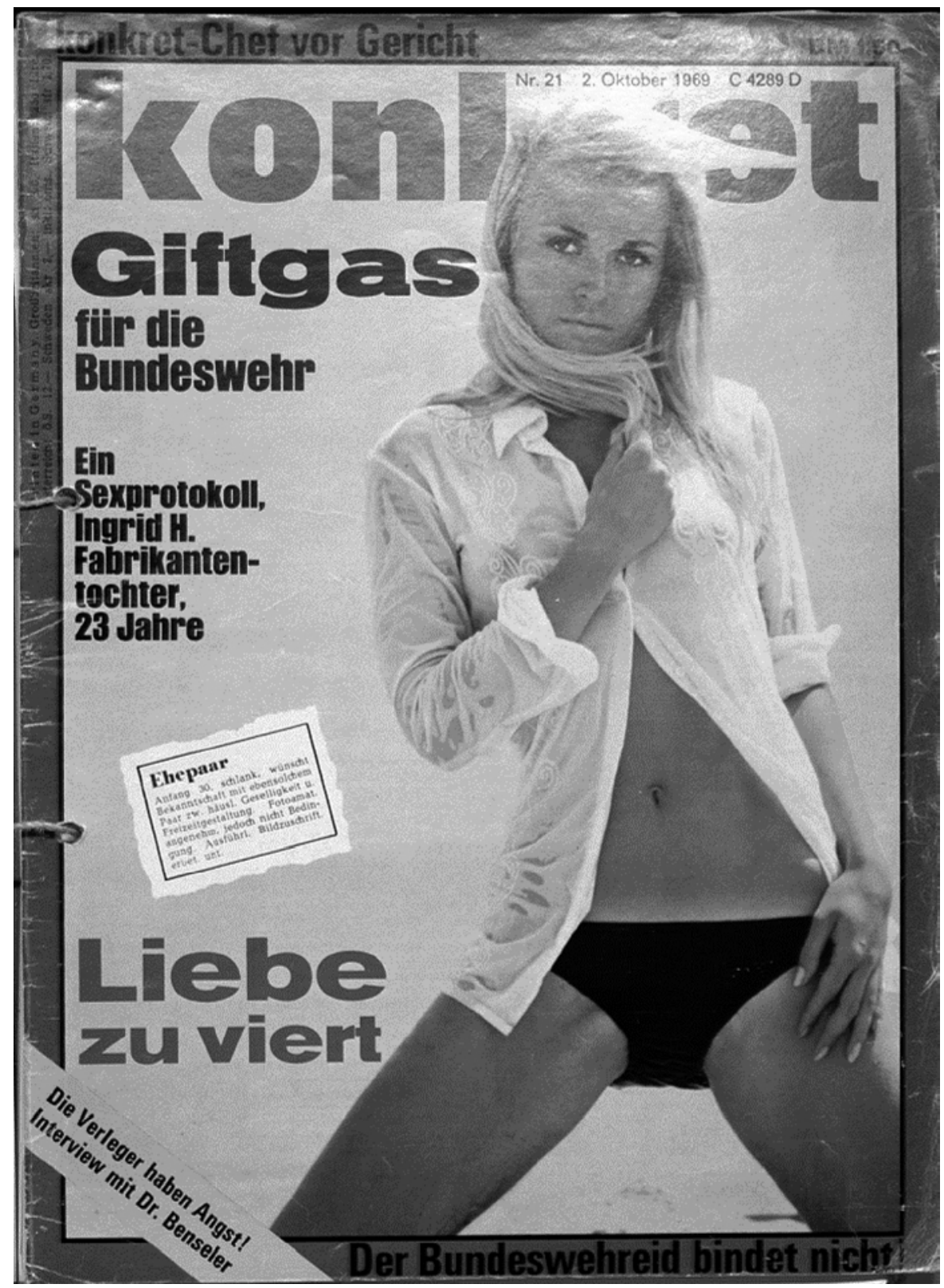

Abb. 2: Titelseite der Zeitschrift konkret Nr. 21 (2.10.1969).

Alle Rechte vorbehalten (Kvv Konkret Vertriebsgesellschaft Für Druck und Andere Medien GmbH \& Co. KG), Quelle: Landesarchiv Schleswig-Holstein, Abt. 811, Nr. 21094. 
sowie „zivilrechtliche[...] Schritte [...] eingeleitet worden“ seien. ${ }^{5}$ Aus der Pressemitteilung ging weiter hervor, dass das Bundesverteidigungsministerium keine Forschungsaufträge an Professor Gärtner sowie das gesamte Kieler Hygiene-Institut erteilt habe. $\underline{6}$

Am 30. Oktober publizierte die konkret in ihrer aktuellen Ausgabe den Artikel „Der Giftmischer von Kiel. Folgen einer Reportage“, in dem die Ereignisse der vertraulichen Sitzung des Kieler Senats vom 14. Oktober wiedergegeben wurden.

Am 6. November konnte Professor Gärtner vor dem Landgericht in Kiel eine einstweilige Verfügung gegen die konkret-Journalisten erwirken. ${ }^{?}$ Daraufhin veröffentlichte der Redakteur der konkret Klaus Rainer Röhl am 13. November einen Widerruf der geäußerten Vorwürfe gegen Professor Gärtner („Prof. Gärtner ist kein Giftmischer“). Der Redakteur wich allerdings nicht von seinen Unterstellungen ab und kündigte weitere Aktionen im Besonderen gegen die Bundeswehr an.

Erst im November 1972 nahm die Horst Gärtner-Affäre schließlich ihr Ende. Sowohl die konkret-Journalisten - Klaus Rainer Röhl, Günter Wallraff und Reinhard Strecker - als auch der nun ehemalige Kieler AStA-Vorsitzende Schlinke wurden zum Widerruf der Beschuldigungen gegen Professor Gärtner verurteilt. Das Urteil gegen die konkret sprach Gärtner eine Veröffentlichung des Urteilsspruchs in den Kieler Nachrichten, der Welt und dem Spiegel zu. $\stackrel{-}{\text { k }}$ konkret-Redakteur Röhl erhielt zudem eine Geldstrafe von 4.000 DM. ${ }^{2}$ Der Kieler Ordinarius Gärtner blieb bis 1979 in seinem Amt.

\section{Ministerialrat Strathmann: Motivation und neue Methoden eines linken Blattes}

Die 1964 neu gegründete Hamburger Zeitschrift konkret machte mit ihrem lasziven Layout die Jugend auf sich aufmerksam. Freizügige Frauen räkelten sich auf den Titelblättern, im Innern wurde rund um das Thema Sexualität aufgeklärt. $\stackrel{10}{ }$ Neben Artikeln zur Anti-Babypille erschienen auch provokative Reportagen über die politischen und gesellschaftlichen Verhältnisse in der Bundesrepublik.

Dies wird an dem Beispiel der Horst Gärtner-Affäre deutlich. Die Reportage „Giftgas für die Bundeswehr“ und der folgende Bericht „Umfrage unter Giftmischern“, die beide am 2. Oktober 1969 in Heft Nr. 21 der konkret veröffentlicht wurden, beschäftigen sich mit einem der Hauptthemen des linken Blattes: der Bundeswehr. Die drei genannten Verfasser des provokativen Artikels - Wallraff, Strecker und Manfred Gfellschild 11 - behaupteten darin, die Bundeswehr beziehungsweise das Bundesverteidigungsministerium vergebe Forschungsaufträge zur Entwicklung von bakteriologischen und chemischen Waffen, kurz B- und C-Waffen, innerhalb der Bundesrepublik 
Deutschland. ${ }^{12}$ So seien über 80 universitäre Institute in der BRD an der B- und C-Waffenforschung beteiligt. Die Bundeswehr arbeite zudem eng mit der Fraunhofer-Gesellschaft zur Förderung der angewandten Forschung e.V. sowie dem Chemiekonzern Bayer A.G. in Leverkusen zusammen, die als unverdächtige Zwischeninstanzen einge-

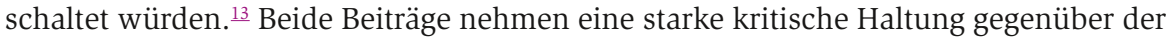
Bundeswehr ein, die sich unter anderem aus dem Protest gegen die am 30. Mai 1968 verabschiedete Notstandsgesetzgebung speiste.

Diese neue Gesetzgebung machte eine Beschneidung der Grundgesetze während eines Ausnahmezustandes möglich. $\stackrel{14}{ }$ Die studentische Protestbewegung sah die Demokratie in der Bundesrepublik hierdurch gefährdet und befürchtete, das Militär könnte für eine erneute diktatorische Machtübernahme eingesetzt werden, wie es bereits 1933 geschehen war. In den Augen der Hamburger Zeitschrift konkret blieb die Befürchtung, die Regierung könne die Bundeswehr nochmals für kriegerische Zwecke missbrauchen, bestehen. In einem Widerruf des Redakteurs zu den geäußerten Vorwürfen gegen Professor Gärtner wird dies deutlich. Die Journalisten hatten der Bundeswehr und allen, „die die Bundeswehr zu einem offensiven Instrument nach außen, insbesondere gegen die DDR und die Sowjetunion, und nach innen gegen die Außerparlamentarische Opposition und die streikenden Arbeiter machen wollen “,,$\frac{15}{\text { den }}$ Kampf angesagt.

Aus dem eben genannten Zitat sticht besonders die Verbindung zur Deutschen Demokratischen Republik (DDR) und zur Sowjetunion hervor. Im Rahmen der Horst Gärtner-Affäre wird bereits in der Reportage „Giftgas für die Bundeswehr“ der Fokus auf Ost-Berlin gelenkt. Ein ehemaliger westdeutscher Wissenschaftler sei nach Ost-Berlin geflüchtet und habe behauptet, ,in der Bundesrepublik [werde] an chemischen und bakteriologischen Kampfmitteln gearbeitet“. $\underline{\text { " }}$ Der Wissenschaftler, auf den die konkret-Journalisten anspielen, war Ehrenfried Petras, der Ende des Jahres 1968 eine Erklärung über seine Kenntnisse zur B- und C-Waffenforschung in Westdeutschland abgab. ${ }^{17}$ Die DDR beziehungsweise die Sozialistische Einheitspartei Deutschlands (SED) nährte die westdeutsche Protestbewegung insbesondere mit Informationen zur nationalsozialistischen Vergangenheit führender Politiker wie dem Bundespräsidenten Heinrich Lübke (1959-1969) und versuchte so, die Bundesrepublik auf allen Ebenen von Politik und Gesellschaft zu diskreditieren. $\frac{18}{}$ Mit derlei Informationen zur Kriegsforschung an westdeutschen Universitäten suchte das kommunistische Berlin, die Bevölkerung auf der anderen Seite der Mauer gezielt in Aufruhr zu versetzen. Dass sich die Zeitschrift konkret auf die DDR berief, ist nicht verwunderlich. Lange Zeit wurde das linke Blatt von der Kommunistischen Partei Deutschlands (KPD) nicht nur mit

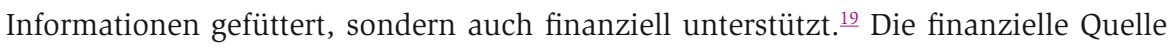
versiegte Mitte der 1960er-Jahre. Laut Frederik Obermaier habe sich die konkret- 


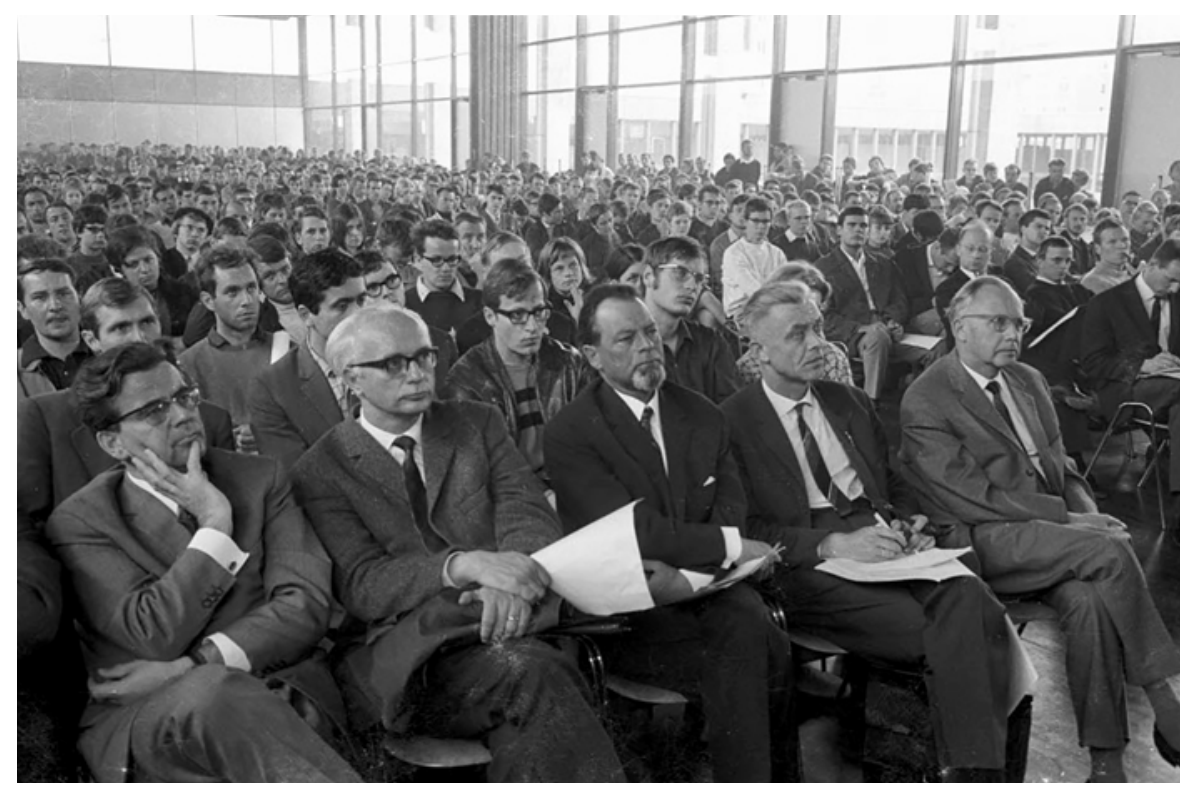

Abb. 3: Streik an der CAU gegen die Notstandsgesetzgebung (29.5.1968).

(C) 1968 Friedrich Magnussen, veröffentlicht unter der Lizenz CC-BY-SA 3.0 DE, Stadtarchiv Kiel, Sign. 21.564.

Redaktion „von dem vermehrt unmenschlich angesehenen System der DDR, das seine eigenen Bürger einsperrt“, $\underline{20}$ distanziert, was die Einstellung der Zahlungen zur Folge hatte. Trotz dessen bestanden - wie nicht nur aus der Reportage gegen die Bundeswehr ersichtlich wird - weiterhin Kontakte in den Osten. Im Falle der Horst Gärtner-Affäre hatte neben den fingierten Telefongesprächen eine Propagandabroschüre der DDR als Recherchematerial für die Beschuldigungen gedient. 21

Da es in Zeiten der Großen Koalition an einer parlamentarischen Opposition fehlte, wurde die konkret quasi das Zentralorgan der Außerparlamentarischen Opposition (APO) .22 Der Protest gegen die Regierung und die Kritik an der Bundeswehr entsprach laut des Redakteurs Röhl den selbstauferlegten genuinen Aufgaben des Blattes:

„Wir werden in Zukunft weitere Dokumente und Artikel über die B- und C-Waffen-Forschung in der Bundesrepublik veröffentlichen, weiß Gott nicht mit dem Ziel, Herrn Prof. Gärtner oder irgendeinen anderen seiner Kollegen persönlich zu diffamieren, zu kränken, zu verleumden oder in seinem Berufsansehen zu schädigen, sondern einzig mit der Absicht, endgültig festzustellen, ob entgegen den der Bundesrepublik auferlegten Verboten Kriegsforschung an 


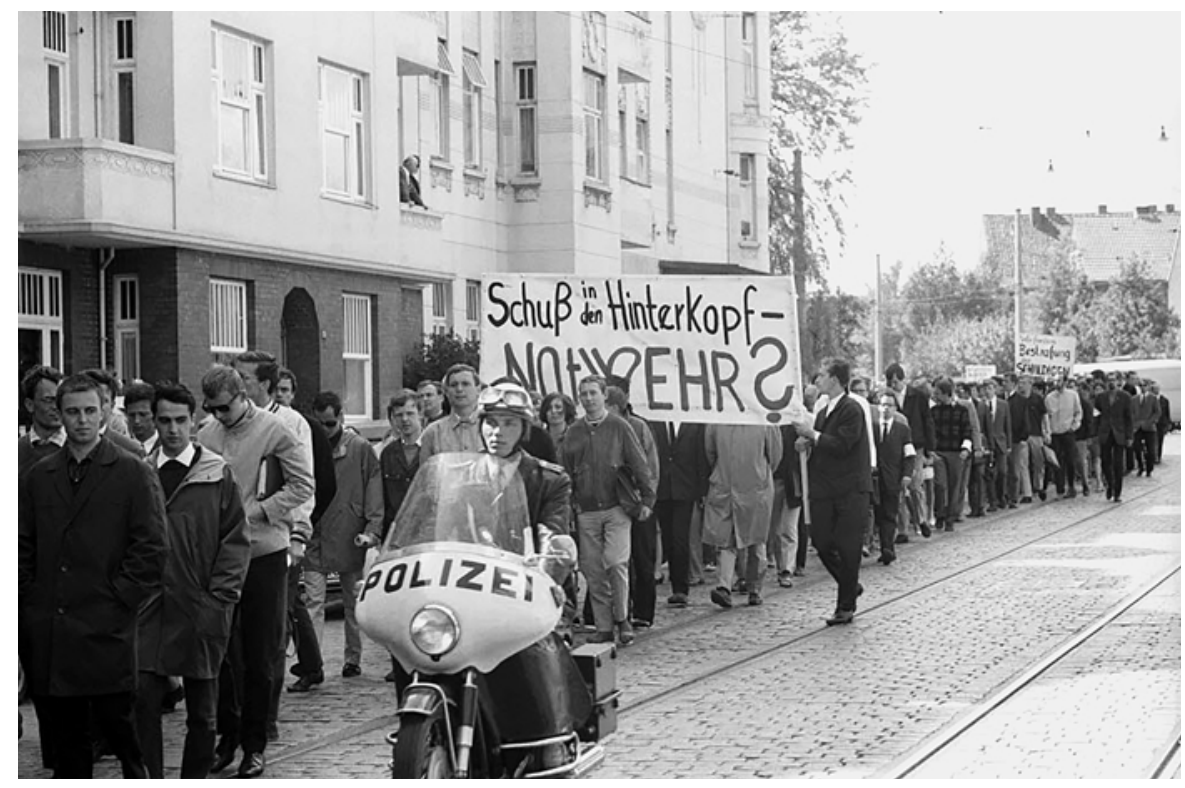

Abb. 4: Studierende der CAU protestieren gegen den Tod des Berliner Kommilitonen Benno Ohnesorg (5.6.1967). (C) 1967 Friedrich Magnussen, veröffentlicht unter der Lizenz CC-BY-SA 3.0 DE, Stadtarchiv Kiel, Sign. 19.035.

A-, B- und C-Waffen betrieben wird, und wenn ja, sie rückhaltlos aufzudecken. “를

Weitere Aspekte, die die konkret zu einer solchen radikalen Reportage bewegten, waren der darin erwähnte Vietnamkrieg und die damit verbundene Kritik an der Außenpolitik der Vereinigten Staaten von Amerika. ${ }^{24}$ Wie viele der Ereignisse in der Bundesrepublik - zum Beispiel der Schuss eines deutschen Polizisten auf den Germanistikstudenten Benno Ohnesorg oder das Attentat auf das Mitglied des Sozialistischen Deutschen Studentenbundes (SDS) und den konkret-Kolumnisten Rudi Dutschke und in der Welt trug auch der Vietnamkrieg zur Radikalisierung der Studentenbewegung bei, die in der zunehmenden Anwendung von Gewalt deutlich wird. $\underline{25}$

Der Einsatz von ABC-Waffen in Südvietnam diente der konkret als ein Paradebeispiel, für welche Zwecke chemische Kampfmittel eingesetzt werden könnten. Es wird deutlich, dass die Redakteure mit den Bildern, die sie durch ihre Reportage erzeugten, das Misstrauen gegenüber der Regierung, dem Establishment und den Professoren sowie die Furcht vor einem Missbrauch von Macht durch das Bundesverteidigungsministerium vertiefen wollten. Eine angebliche Geheimhaltung solcher Forschungs- 


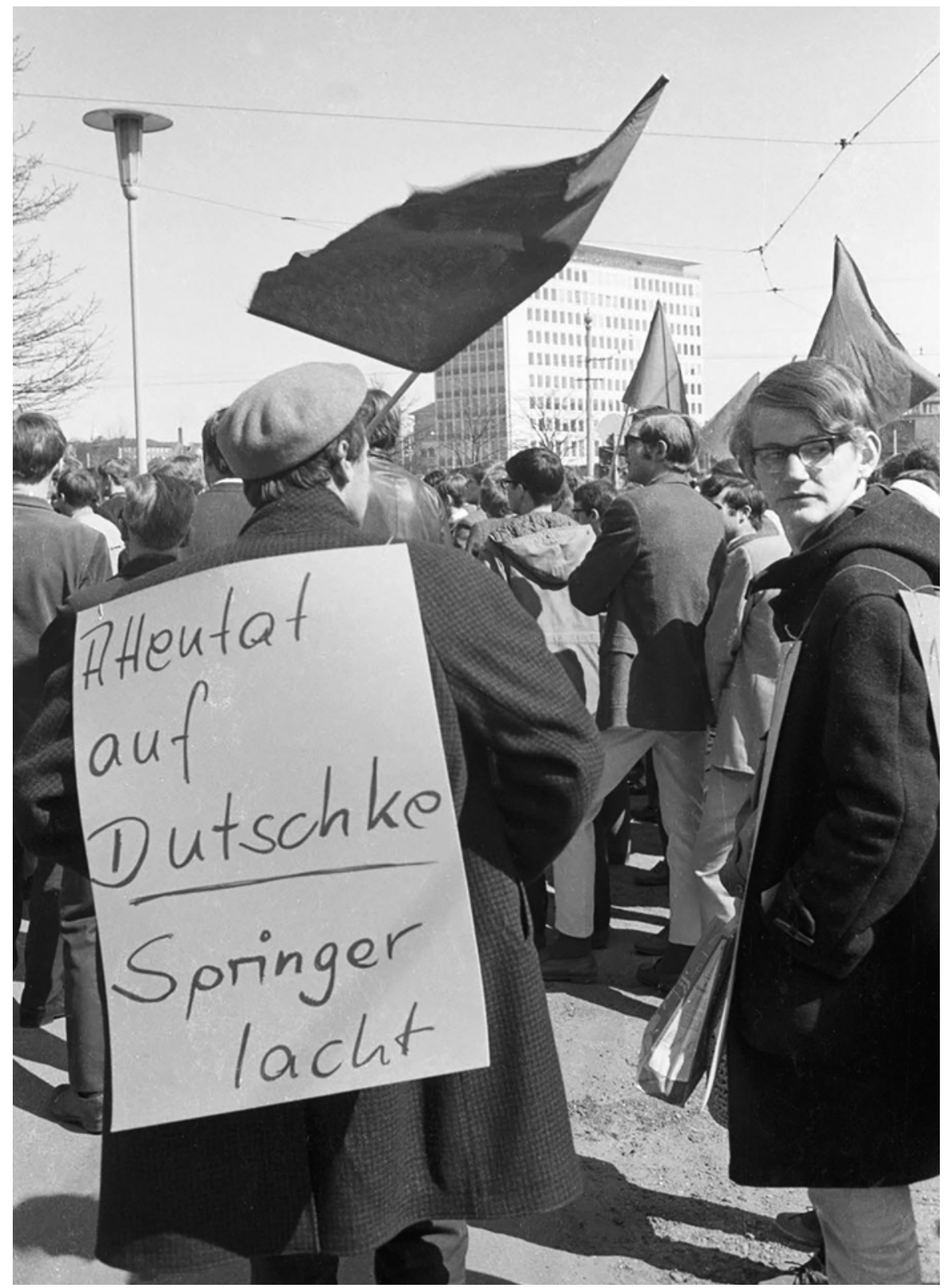

Abb. 5: Studierende demonstrieren gegen Attentat auf Rudi Dutschke auf dem Rathausplatz (13.4.1968). (C) 1968 Friedrich Magnussen, veröffentlicht unter der Lizenz CC-BY-SA 3.0 DE, Stadtarchiv Kiel, Sign. 21.488. 
aufträge, die die ganze Bundesrepublik in einen erneuten Krieg hätte führen können, war in den Augen der 68er ein Riesenskandal.

In dem an die Reportage anschließenden Bericht mit dem Titel „Umfrage bei Giftmischern" wurden Interviews mit vier Wissenschaftlern aus Deutschland abgedruckt, die aufgrund fingierter Telefonanrufe entstanden waren. Neben Professor Horst Gärtner erhielten Professor Helmut Kewitz (Freie Universität Berlin), Professor Otto Klimmer (Universität Bonn) und Professor Hermann Eyer (Universität München) solche Anrufe. Der Journalist Günter Wallraff gab sich als Ministerialrat Strathmann aus, der zu dieser Zeit Referent des Bundesverteidigungsministeriums war. $\underline{26}$

Allen vier Ordinarien wurde in dem Bericht und aufgrund der fingierten Anrufe vorgeworfen, an B- und C-Waffen mitzuarbeiten oder das Interesse an einem solchen Forschungsprojekt zu haben. ${ }^{27}$ Die Kritik an Regierung, Bundeswehr und den Gräueln von chemischen Waffen vermischten sich in dem Beitrag mit einer weiteren treibenden Kraft der 68er-Bewegung, nämlich mit der Kritik an der nicht aufgearbeiteten NS-Vergangenheit von weiten Teilen der deutschen Gesellschaft - so auch von Professoren, führenden Politikern und des gesamten Establishments. ${ }^{28}$ Die nationalsozialistische Vergangenheit der Professoren Klimmer und Eyer wurde im konkret-Bericht in einen direkten Zusammenhang zu den angeblichen Forschungsprojekten für chemische Waffen gesetzt. So heißt es über Eyer, dass auch er „in der Lage [sei], ,Füh-

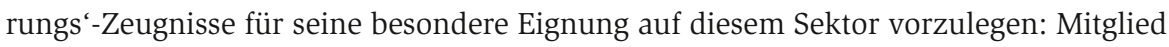
der SA seit 1933, der NSDAP seit 1935, 1939-1945 Leiter des Fleckfieber- und Virusforschungsinstituts des O.K.H. in Krakau“.르 Diese Argumentation suggeriert, dass jene Professoren, die in der einen oder anderen Weise in das NS-Regime eingebunden waren, an Projekten, die einen erneuten Krieg auslösen könnten, interessiert sein mussten. Das kollektive Schweigen über die nationalsozialistische Vergangenheit hochrangiger Vertreter in den Institutionen oder der Regierung war ein rotes Tuch für die studentische Protestbewegung. Was zunächst als Faschismusdebatte begann, „reduzierte sich [...] innerhalb der sozialistischen Linken der Jahre 1968ff sehr bald auf eine Nationalsozialismusdebatte“. .30 Auch an den Hochschulen kam es zunehmend zu Protestaktionen gegen die Ordinarien, deren braune Vergangenheit öffentlich gemacht wurde. Die Studierenden setzten sich „auf das hohe Roß des Anklägers“, $\underline{\text {, }}$ wie es Hans-Ulrich Thamer treffend formuliert. Die 68er-Generation, die nicht durch das NS-Regime sozialisiert worden war, distanzierte sich immer mehr von der Nachkriegsund Aufbaugeneration beziehungsweise von ihren Eltern, die die NS-Diktatur und den Zweiten Weltkrieg miterlebt und teilweise mitgetragen hatten. ${ }^{32}$ Zusätzlich wurde dieser Konflikt zwischen den beiden Generationen durch den Einfluss der DDR, die „sich selbst als Hort des Antifaschismus“ begriff, $\underline{\underline{33}}$ geschürt, wie bereits am Beispiel Heinrich Lübkes gezeigt wurde. 
Aus dem Vorangegangenen werden einige der Gründe ersichtlich, die die Zeitschrift konkret dazu bewegten, die Reportage „Giftgas für die Bundeswehr“ zu verfassen. Gleichzeitig fungierten derlei Berichterstattungen als Antriebskraft einer bundesdeutschen Studentenbewegung für ihre Proteste gegen die Bonner Regierung und einen angeblichen Verfall der Demokratie. Aus der Reportage selbst sowie dem Verhalten der konkret-Redaktion wird ersichtlich, dass zeitgleich zu der steigenden Radikalisierung innerhalb der bundesdeutschen Studentenbewegung auch die konkret-Journalisten eine extremere Vorgehensweise in Bezug auf ihren Schreib- und Reportagestil anwandten. Die Zuspitzung dieser gesamtgesellschaftlichen Entwicklungen sollte schließlich in den 1970er Jahren kommunistische Akteursgruppen sowie den linksextremistischen Terrorismus hervorbringen.

Das linke Blatt verwendete eine für die damalige Zeit neuartige Methode des Journalismus, die als New Journalism bezeichnet wird. Das bedeutet, dass die reine Berichterstattung zunehmend vernachlässigt wurde und es zum Ergreifen von Partei sowie Einnehmen einer aktiven Rolle seitens der konkret-Journalisten kam. ${ }^{34}$

Die Anschuldigungen aus der Reportage beruhten, wie bereits erwähnt, lediglich auf den Informationen der Propagandabroschüre der DDR. $\stackrel{35}{5}$ Stichhaltige Beweise oder belastbare Quellen fehlten, weswegen vonseiten der konkret-Redaktion wohl auf den Einsatz fingierter Telefonanrufe zurückgegriffen wurde. Innerhalb des Prozesszeitraumes nahm die konkret bei der Berichterstattung, die lediglich bis zum Erscheinen des urteilsgemäßen Widerrufs in dem linken Blatt am 13. November 1969 anhielt, eine aktive und subjektive Rolle ein, indem sie durch weitere Beiträge in der Zeitschrift fortwährend auf den aufgestellten Behauptungen beharrte und diese weiter verteidigte. In ihren Reportagen wurden die Inhalte der Telefongespräche ferner nicht dem ursprünglichen Wortlaut entsprechend wiedergegeben und mit eigenen Behauptungen und Schlussfolgerungen unterfüttert, die unzureichend belegt worden waren. So wurde das Bild vermittelt, dass alle Professoren dem fingierten Auftrag des Bundesverteidigungsministeriums zugestimmt hätten. In ähnlicher Weise verfuhren die konkret-Journalisten mit dem Frankfurter Experten und Virologen Oswin Günther - seinerzeit wissenschaftliches Mitglied der Paul-Ehrlich-Stiftung. Dessen Fachwissen zur Überwindung der Immunitätsbarriere für Pockenerreger wurde in einem Folgeartikel dazu genutzt, um eine Aussage Professor Gärtners während der Senatssitzung vom 14. Oktober 1969 zu den Immunitätsbarrieren für Pockenerreger zu widerlegen. $\frac{36}{6}$ Wie in den Fällen davor wurde Günthers Aussage am Telefon dazu benutzt, nicht nur die anderen Wissenschaftler, sondern auch die Bonner Regierung zu diskreditieren.

Besagter Folgeartikel „Der Giftmischer von Kiel. Folgen einer Reportage“ entstand nach einer Sitzung des Senats der Christian-Albrechts-Universität zu Kiel (CAU), die heimlich mit einem Tonbandgerät aufgenommen worden war. ${ }^{37}$ Dem Artikel zufolge 
habe „einer der an der Sitzung teilnehmenden Professoren [das vertrauliche Protokoll der Senatssitzung] zur Verfügung“ gestellt. $\frac{38}{}$ Wieder wurde auf die objektive Berichterstattung verzichtet, das Gesprochene unvollständig und sinnentstellt wiedergegeben, und die einzelnen Abschnitte wurden mit eigenen Behauptungen und Schlussfolgerungen versehen. Das Rektorat der CAU nahm in der sozialdemokratischen Nordwoche zu der Behauptung, Professoren hätten die Tonbandaufnahme weitergeleitet, Stellung. Demzufolge hätten alle „beiwohnenden Professoren eine verbindliche Erklärung abgegeben “" $\underline{\underline{39}}$ in der sie bestritten, in irgendeiner Weise mit diesen Aufnahmen oder der konkret in Verbindung zu stehen. In dem besagten Artikel wurde nicht nur Professor Gärtner auf die Anklagebank gesetzt, sondern auch Professor Heinz Albrecht Lüllmann, der zu diesem Zeitpunkt ordentlicher Professor am Pharmakologischen Institut und Dekan der Medizinischen Fakultät war. ${ }^{40}$ Dieser wurde ebenfalls beschuldigt, „im Auftrage des Bundesverteidigungsministeriums“ zu arbeiten. sich herausstellte, arbeitete Lüllmann an der Erforschung von Gegengiften für Organophosphate. ${ }^{42}$ Dass dieser Forschungsbereich vom Bundesverteidigungsministerium finanziert wurde, stimmte nur zum Teil. Lüllmann hatte um Mittelzuweisungen gebeten, um Personal, Geräte und Betriebsmittel aufzustocken. $\stackrel{43}{ }$ Die Vertreter der Kieler Studierenden, die an der Senatssitzung teilnahmen, wurden in dem Artikel als Ankläger oder sogar Richter dargestellt, die die Professoren Gärtner und Lüllmann ins Kreuzverhör nahmen. Das Rektorat der Kieler Universität entschärfte die Anschuldigungen gegen Lüllmann mit einer Stellungnahme. Hierin hieß es, dass bereits im Sommersemester 1969 studentische Vertreter die Räumlichkeiten Lüllmanns besichtigt hätten, um zu klären, dass der Ordinarius nicht wie behauptet „an Experimenten mit dem hochtoxischen Nervengas Soman“ $\underline{44}$ für das Bundesverteidigungsministerium arbeite, sondern lediglich Gegengifte erforsche. Lüllmann sei zudem dazu bereit gewesen, dass „die Senatskommission sein Institut jederzeit kontrollieren [könne]“. “5ㅡ

Am Beispiel der Zeitschrift konkret ist deutlich zu erkennen, dass die 68er-Bewegung sich das Medium Zeitung oder allgemein die Massenmedien zu eigen gemacht hat. Um die Gesellschaft in Aufruhr zu versetzen, bedienten sich die konkret-Journalisten nicht nur anklagender und diskreditierender Worte gegen Regierung, Bundeswehr und Ordinarien. Sie untermalten ihre provokanten Texte auch mit schockierenden Bildern, auf denen Menschen abgebildet waren, die beispielsweise mit dem Nervengift Soman in Berührung gekommen und durch dessen toxische Wirkung entstellt worden waren. $\frac{46}{}$ Sie arbeiteten zudem - wie bereits dargestellt - mit fingierten Beweisen und im Stil des New Journalism, durch die es ihnen erst gelang, größere Massen aufzurütteln und sich womöglich kritischer mit gesellschaftlichen wie politischen Themen auseinanderzusetzen. Denn solche produzierten Bilder riefen beim Rezipienten unweigerlich Emotionen hervor und führten, wie es im weiteren Verlauf 
bei den Studierenden der Christiana Albertina zu sehen sein wird, gerade bei einer Massenbewegung wie den 68ern zu Empörung und Protest.

\section{Von der konkret zum AStA-info: Der Protest weitet sich aus}

Die Kieler Studentenbewegung folgte dem Vorbild aus den Großstädten der Bundesrepublik wie Berlin, Frankfurt, Hamburg und Westberlin. Denn auch ihr Verhalten wurde durch die gesellschaftlichen und politischen Ereignisse beeinflusst, wie am Beispiel der Zeitschrift konkret bereits gezeigt werden konnte. Der Aktionismus der antiautoritären Bewegung sorgte in Kiel durchaus für größere Unruhen, ${ }^{47}$ bei denen es auch zur Anwendung von Gewalt kam, und führte hier im Zuge der Radikalisierung sowohl zur Bildung von kommunistischen Gruppierungen als auch zum Anschluss

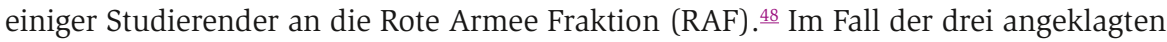
Mitarbeiter des AStA - Manfred Jungjohann, Dietmar Schlinke und Anthon Schmelzer -, die wegen des Beitrags „Kriegsforschung in Kiel oder Gärtner muß Gärtner werden“ im AStA-info in Kiel vor Gericht standen, traten Beweggründe wie der Vietnam-

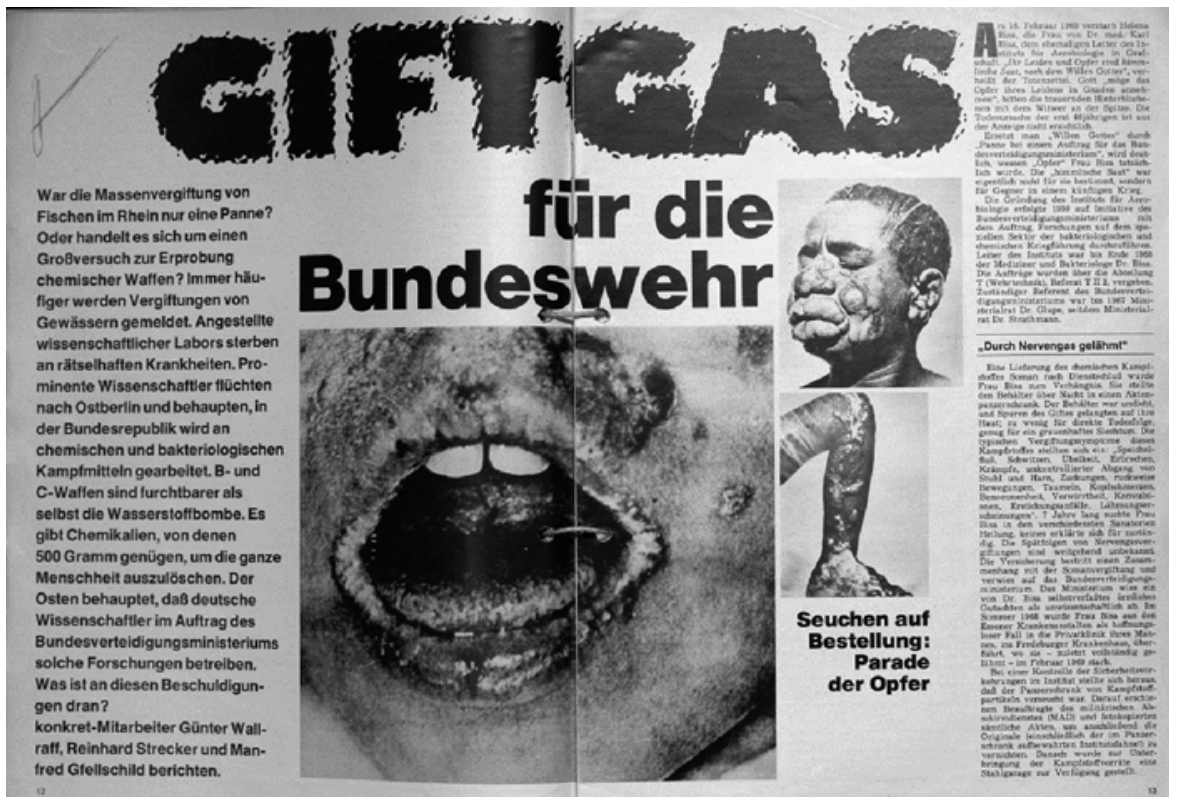

Abb. 6: Artikel ,Giftgas für die Bundeswehr' aus konkret Nr. 21 (2.10.1969).

Alle Rechte vorbehalten (Kvv Konkret Vertriebsgesellschaft Für Druck und Andere Medien GmbH \& Co. KG), Quelle: Landesarchiv Schleswig-Holstein, Abt. 811, Nr. 21094. 

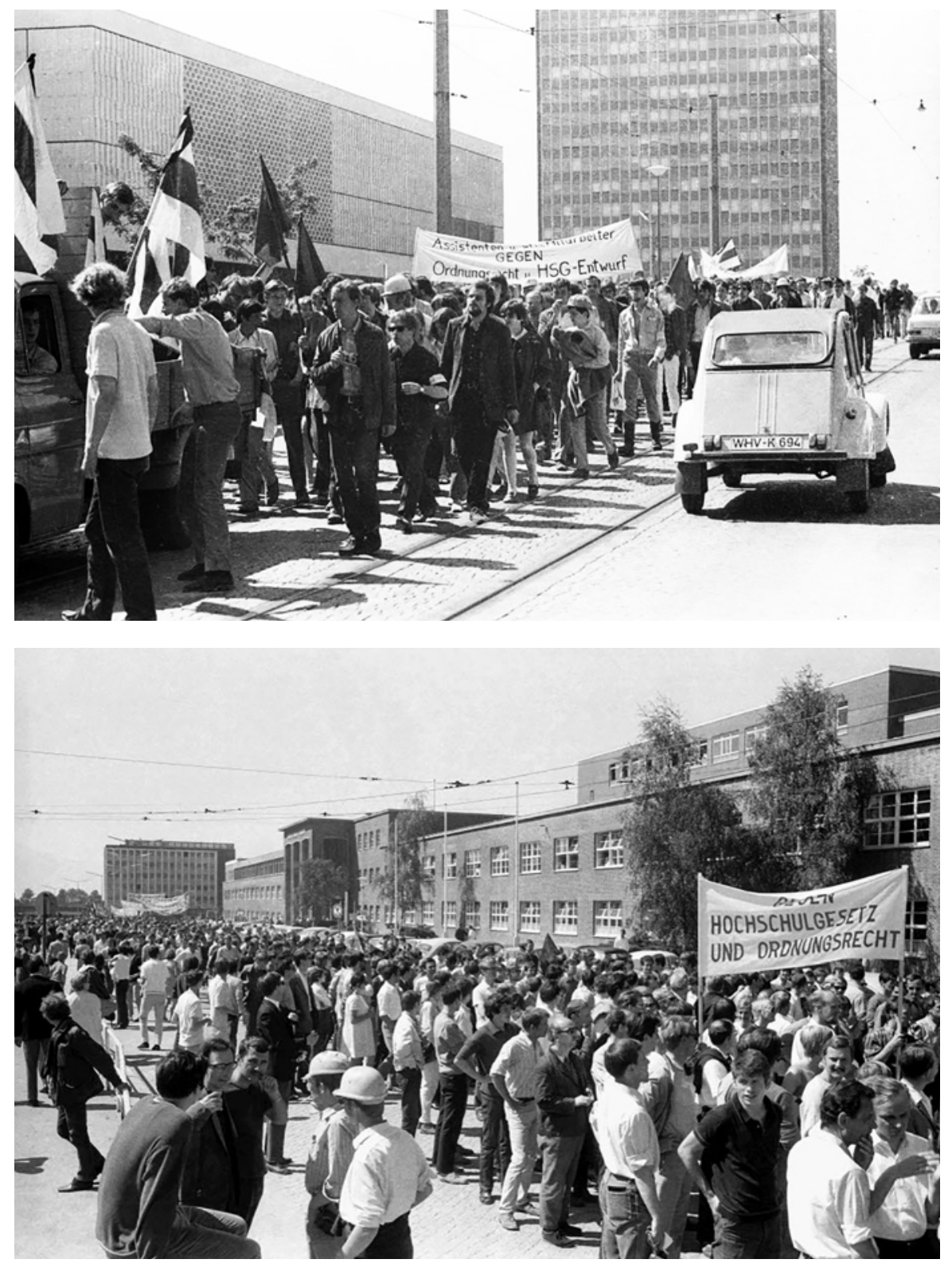

Abb. 7 und 8: Demonstrationen gegen neues Hochschulgesetz und Ordnungsrecht in der Holtenauer Straße (9.6.1969).

(c) 1969 Friedrich Magnussen, veröffentlicht unter der Lizenz CC-BY-SA 3.0 DE, Stadtarchiv Kiel, Sign. 21.884 und 21.900. 
krieg, die Große Koalition und die Notstandsgesetzgebung, die für die Redaktion der konkret gewichtige Einflüsse darstellten, in den Hintergrund. Vielmehr überschattete die noch ausstehende demokratische Hochschulreform in Kiel alle anderen die Bewegung antreibenden Ereignisse. $\underline{49}$

So reagierten die Kieler Studierenden nicht nur mit einem Artikel in dem studentischen Informationsblatt AStA-info auf die Reportage der linken Hamburger Zeitschrift, sondern hatten auch die Möglichkeit, vor Ort an der CAU mit weiteren Aktionsformen zu agieren. Einerseits nutzten sie die Senatssitzung, um Professor Gärtner persönlich und vor den Augen seiner Kollegen zu diffamieren. Studierende besetzten andererseits dessen Büro sowie weitere Teile der Kieler Hochschule. $\underline{50}$

In ihrem Artikel „Kriegsforschung in Kiel oder Gärtner muß Gärtner werden“ aus dem AStA-info-Heft Nr. 57 verlangten die Autoren, dass „das, was sich im ,HygieneInstitut' abgespielt hat, sofort an die Öffentlichkeit [muß] “, sowie die Kontrolle wissenschaftlicher Projekte wie solche, die Professor Gärtner betrieben haben sollte. ${ }^{51}$ Der Artikel warnte vor dem möglichen Einsatz von Bakterien als Offensiv-Waffe zur Vernichtung ganzer Völker, gleichzeitig wurde die Politisierung von Forschung und Wissenschaft kritisiert.

Darüber hinaus erklärten die beiden studentischen Senatsvertreter Schlinke und Stabel in dem Folgeartikel „Der Giftmischer von Kiel“ den konkret-Journalisten, dass sie jahrelang versucht hätten, „,in allen Kommissionen dieser Universität einen Zugang

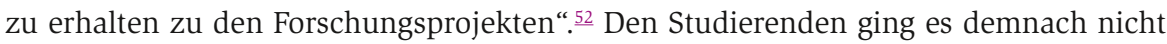
vorrangig um die gesellschaftlichen und transnationalen Faktoren, die innerhalb der 68er-Bewegung rege diskutiert und innerhalb der konkret-Berichterstattung bedient wurden. Sie machten hiermit vielmehr auf die ungenügende Mitbestimmung der Studentenschaft und die fehlende Transparenz der sogenannten Ordinarienuniversität aufmerksam. Diese Mängel sollten mit einer Reform des bisher bestehenden Hochschulsystems aufgehoben werden, die in Hessen bereits 1966 auf gesetzlichem Weg umgesetzt worden war. $\frac{53}{}$ In den 1960er-Jahren herrschten an den Universitäten zumeist weiterhin die traditionellen hierarchischen Strukturen mit Ritualen wie dem der Rektoratsübergabe, die in den mittelalterlichen Talaren vollzogen wurde. $\frac{54}{4}$ Anhand der Aktionsform des sogenannten Muff-outs, die sie von den Hamburger Studierenden adaptierten, zeigten die Kieler Studierenden im Mai 1968 beispielsweise ihr Missfallen gegenüber diesem traditionellen Akt und zogen das Ritual ins Lächerliche. $\frac{55}{5}$ Hierbei kam nicht nur die Parole „Unter den Talaren - Muff von 1000 Jahren“ zum Einsatz, sondern auch eine Parodie der Rektoratsübergabe mit Klobürste als Zepter und Stahlhelm. $\frac{56}{}$

Mit solchen Aktionen versuchten die Protestierenden auf die Notwendigkeit einer Demokratisierung der Universitäten aufmerksam zu machen. Der SDS ging auf bun- 


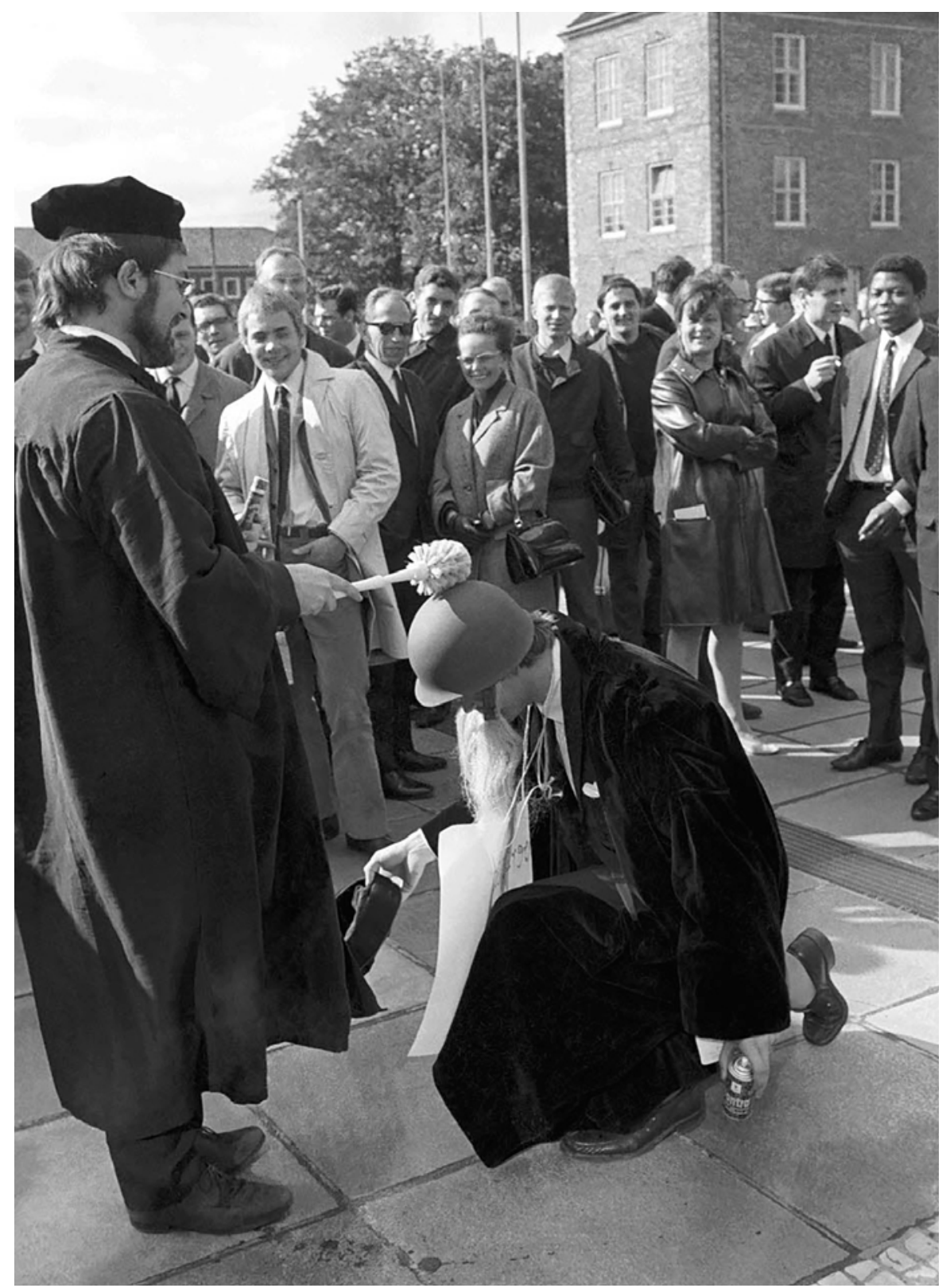

Abb. 9: Studentisches Sit-in zur Rektoratsübergabe der CAU auf dem Schlossplatz (14.5.1968).

(c) 1968 Friedrich Magnussen, veröffentlicht unter der Lizenz CC-BY-SA 3.0 DE,

Stadtarchiv Kiel, Sign. 21.548 


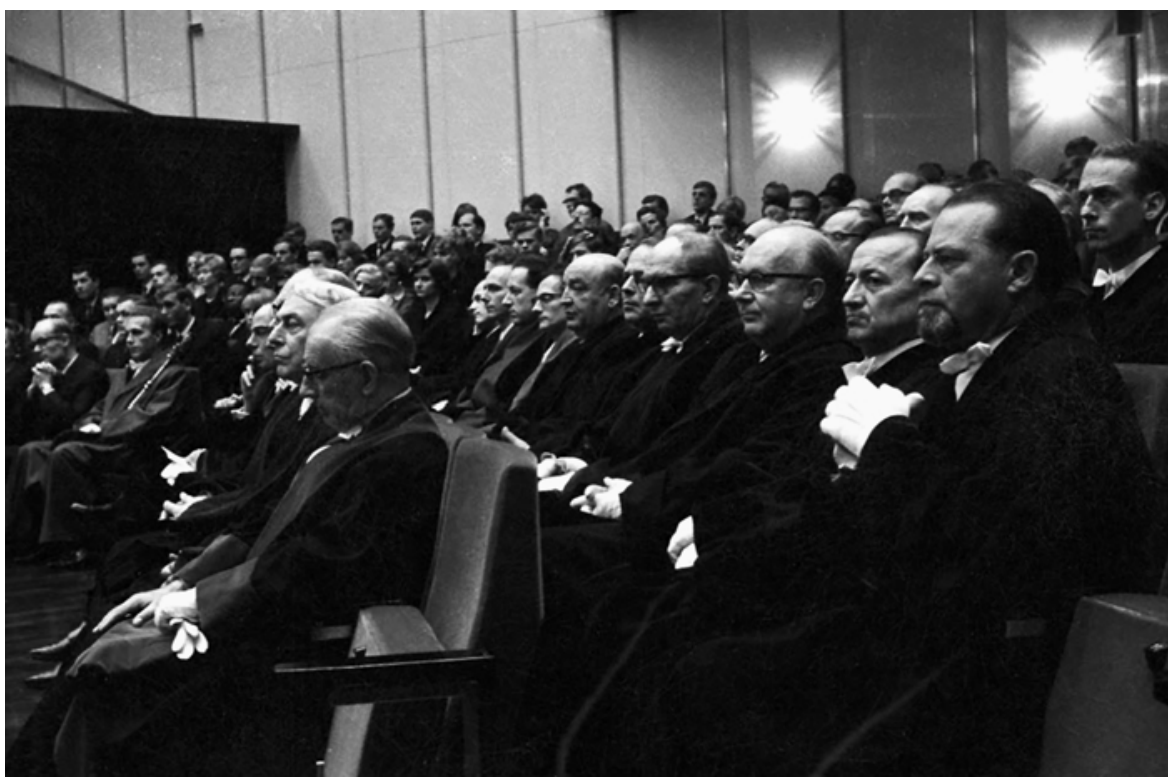

Abb. 10: Feierliche Verpflichtung der neu immatrikulierten Studierenden der CAU im Konzertsaal im Schloss (18.11.1966). (C) 1966 Friedrich Magnussen, veröffentlicht unter der Lizenz CC-BY-SA 3.0 DE, Stadtarchiv Kiel, Sign. 18.995.

desdeutscher Ebene sogar so weit, die Universitätsverwaltung durch ein Rätesystem ersetzen zu wollen,,$\underline{57}$ und weigerte sich seit 1968, an den Diskussionen zu einem neuen Hochschulgesetz teilzunehmen. $\stackrel{58}{ }$ Die Professoren hingegen hatten die Ordinarienuniversität bisher nach ihren eigenen Vorstellungen im Sinne einer Selbstverwaltung leiten können und fürchteten nun einen vermeintlichen Autonomieverlust, der mit einer solchen demokratischen Hochschulreform einherginge. .99 So trafen die Ordinarien sämtliche Entscheidungen innerhalb der Universität, bei denen Assistentinnen und Assistenten sowie Studierende keinerlei Stimmberechtigung erhielten. ${ }^{60}$

Darüber hinaus kam es in den 1960er-Jahren zu einem rapiden Anstieg der Studierendenzahlen, was auf eine höhere Geburtenrate bei den Nachkriegsjahrgängen zurückzuführen ist. .1 Dies stellte die Hochschulen der Bundesrepublik vor eine neue Herausforderung. Das hierarchische Hochschulsystem mit seiner akademischen Selbstverwaltung war nicht dafür ausgelegt, diese Massen an Lernwilligen mit deren zunehmenden Forderungen nach mehr Mitbestimmung und Transparenz zu bewältigen. Auch an der Förde strömten die Massen an die Christiana Albertina, die sich aufgrund des veralteten Systems, welches sie dort erwartete, mit unzureichenden Studienbedingungen konfrontiert sahen. $\underline{62}$ Dass die Kieler Universität mit diesem Ansturm 
überfordert war, zeigte sich beispielsweise im Januar 1968. Obwohl der benötigte Ausbau des Hochschulgeländes und des Personals in den vorangegangenen Jahren stark vorangetrieben worden war, musste die CAU weitere Maßnahmen ergreifen, um der steigenden Studentenzahl entgegenzuwirken. $\frac{63}{3}$ Daher sollte an der Universität unter anderem eine neue Immatrikulationsverordnung eingeführt werden, „die zu einer Zwangsexmatrikulation von Langzeitstudierenden geführt hätte“. $\underline{\text { 4 }}$ Es kam zu einer Demonstration, an der sich mehr als 2.500 Studierende beteiligten.

Auch ein Jahr später hatte sich an den alten Strukturen der CAU kaum etwas verändert, und in Bezug auf die überfällige hochschulpolitische Reform waren keine Fortschritte zu erkennen. Obwohl die Studierenden mehr Mitbestimmung forderten, wirkten sie mit ihren Störaktionen einer neuen Hochschulgesetzgebung eher entgegen. $\frac{65}{}$ Die allgemeine Lage an der Kieler Universität war angespannt. Der Landtag erkannte im April 1969 den Entwurf eines neuen Hochschulrechts mit Ordnungsrecht an, welches allerdings keine Neuordnung des bisherigen Systems mit sich bringen sollte. ${ }^{6}$ Hierdurch sollte nicht nur den Assistenten und Assistentinnen sowie Studierenden die Drittelparität verwehrt bleiben, sondern auch eine „verstärkte staatliche Kontrolle und die Beschneidung der Selbstverwaltung“ in Kraft treten. .7 Dieses neue Gesetz sei laut Stefan Bichow aufgrund der zahlreichen Disziplinarverfahren gegen die antiautoritären Studierenden vom Kultusminister eingeführt worden, um mit dem neuen Ordnungsrecht und den darin enthaltenen Sanktionen gegen weitere Protestaktionen vorgehen zu können. ${ }^{68}$ In diesem Fall standen die Hochschule und die Studierendenschaft ungewöhnlicherweise auf derselben Seite, da sich die repressiven Maßnahmen auf beide Parteien ausgewirkt hätten. Aufgrund verfassungsrechtlicher Bedenken wurde das neue Gesetz letztendlich nicht unterzeichnet. Die Frage nach einer ausstehenden Hochschulreform mit der geforderten Drittelparität und Transparenz des Universitätsgeschehens konnte auch zum Ende der Kieler 68er-Bewegung nicht geklärt werden. Die neue Hochschulgesetzgebung sollte an der Kieler Universität erst im Jahr 1973 in Kraft treten. $\underline{\text { 우 }}$

Daraus lassen sich folgende Erkenntnisse gewinnen, welche Ereignisse den Forderungen - Transparenz und Kontrolle im Bereich der Forschung - der drei Verfasser zugrunde lagen. Das hierarchische System der Kieler Ordinarienuniversität bot keinerlei Transparenz für die Studierendenschaft sowie den Akademischen Mittelbau. Bei Entscheidungen zum Universitätsgeschehen, die die Ordinarien meist unter Ausschluss der Öffentlichkeit hinter verschlossenen Türen diskutierten, wurden sie weiterhin kaum miteinbezogen. Ähnlich wie bei der Notstandsgesetzgebung fürchtete die Kieler Studierendenschaft den Missbrauch der Wissenschaft einerseits durch die Professorenschaft und andererseits durch das Bundesverteidigungsministerium. $\frac{70}{}$ Aufgrund dieser universitären Verhältnisse pochten die studentischen Vertreter Stabel 


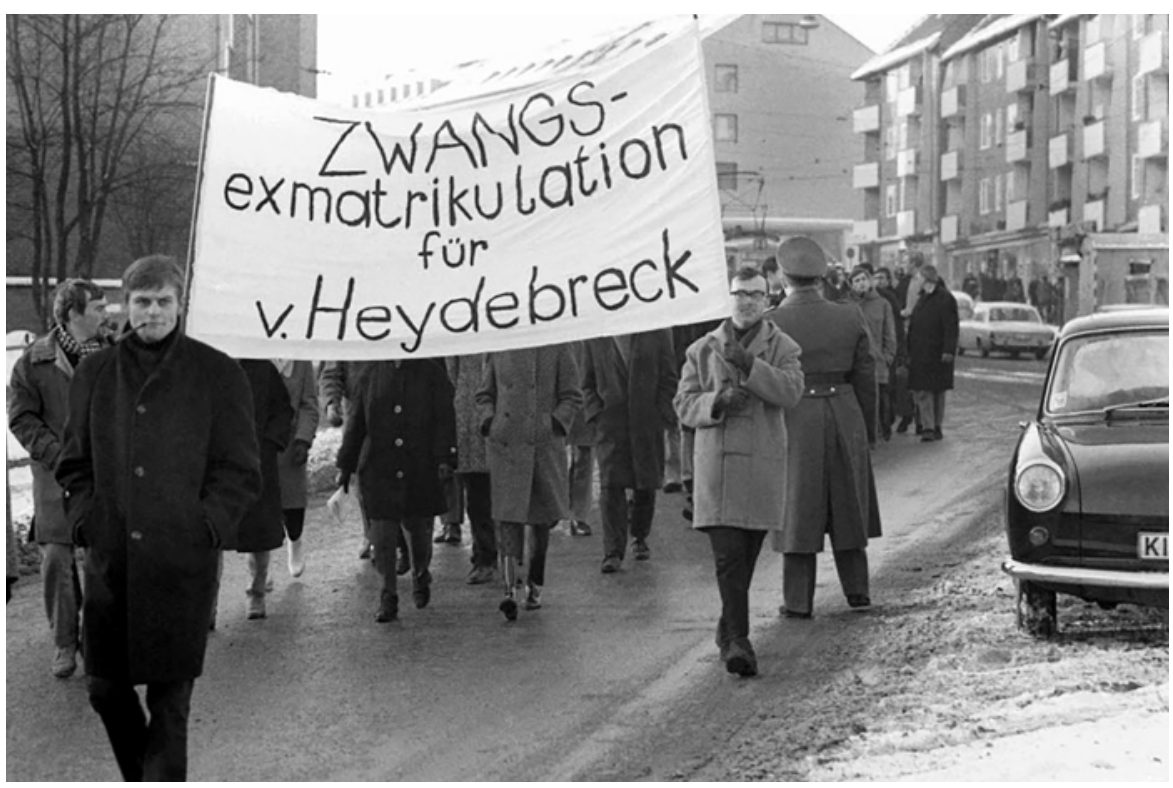

Abb. 11: Kieler Studierende demonstrieren gegen Zwangsexmatrikulation (10.1.1968).

(C) 1968 Friedrich Magnussen, veröffentlicht unter der Lizenz CC-BY-SA 3.0 DE,

Stadtarchiv Kiel, Sign. 21.623.

und Schlinke, wie oben bereits erwähnt wurde, auf die Kontrolle der verschiedenen Forschungsbereiche. Dabei ging es ihnen nicht nur um eine einmalige Besichtigung des Hygiene-Instituts, um festzustellen, ob Professor Gärtner tatsächlich an Forschungsprojekten für die Bundeswehr gearbeitet habe oder immer noch arbeite. Vielmehr lag es in ihrem Interesse, dass Studierende, Assistenten und „andere Kontrollierende an der Universität eine Einsicht in das letzte Material“ bekommen sollten. ${ }^{71}$ Bei dem letzten Material handelt es sich um die Forschungsgelder und -projekte, deren Einsicht der Öffentlichkeit verwehrt wurde. Stabel verlangte, „daß Herr Gärtner so schnell wie möglich entweder einer Kommission dieses Senats oder anderer Institutionen sämtliche Forschungsprojekte auf den Tisch" legen solle, denn bisher seien „sehr, sehr viele Punkte unter den Tisch gefallen“.르 Bereits in der Senatssitzung am 14. Oktober 1969 war auf diese Forderung der studentischen Vertreter eingegangen und eine Untersuchungskommission aus Senatsmitgliedern und einem studentischen Vertreter zusammengestellt worden. Diese sollte das Hygiene-Institut auf etwaige Anhaltspunkte bezüglich der Kriegsforschung untersuchen. Obwohl damit ihre Forderungen zumindest in Hinsicht auf Horst Gärtner erfüllt wurden, verweigerten beide studentischen Vertreter die Mitarbeit bei dieser Kommission. In den Kieler Nachrichten 
erklärte ein AStA-Sprecher, dass „er [...] nichts von einer Überprüfung durch eine Untersuchungskommission [halte], da seiner Meinung nach im Institut bereits alles vertuscht worden sei oder vertuscht werde““ $\underline{\underline{73}}$ Das Rektorat der CAU erklärte in einer Stellungnahme, dass - aufgrund der Verweigerung der Mitarbeit in der Kommission durch den ersten AStA-Vorsitzenden und den Vorsitzenden der Fachschaft - die Arbeit der Kommission aufgegeben werden musste. Denn „[die] Arbeit einer Kommission [...] konnte nur bei Mitarbeit studentischer Vertreter, von denen diese vermeintlichen Behauptungen ungeprüft übernommen worden waren, sinnvoll sein." $\underline{\text { } 74}$

Die Herausgeber und Autoren des AStA-info zeigten in ihrer Vorgehensweise zu Beginn der Horst Gärtner-Affäre ein ähnliches Verhalten wie die konkret-Journalisten. Einzig die Reportage des linken Blattes und ein angebliches Telefongespräch mit konkret-Journalist Günter Wallraff nahmen sie als Grundlage für ihren eigenen Artikel. Weitere Recherchen unterließen sie und übernahmen die Anschuldigungen gegen Professor Gärtner vollkommen unkritisch. ${ }^{25}$

Die Kieler Studierenden gingen allerdings noch weiter als die konkret-Journalisten und bedienten sich weiterer Aktionsformen wie der Störungen an der Universität, um ihre Forderungen durchzusetzen. $\frac{76}{}$ Diese Art von Aktionismus wurde von der Bewegung als Strategie der begrenzten Regelverletzung bezeichnet. Durch das unbefugte Betreten der universitären Räumlichkeiten verstießen die Kieler Studierenden bewusst gegen die Hausordnung der CAU: „Sie [die Akteure der Bewegung] verfolgten die Absicht, nicht den Eindruck eines Gesetzesbruchs, sondern den symbolischen Charakter einer Übertretung bestehender Regeln oder Gesetze hervortreten zu lassen. “그 Mit diesen Aktionsformen sollte die Aufmerksamkeit der Öffentlichkeit erlangt und verdeutlicht werden, „daß es ihnen Ernst mit der Regelverletzung sei“. .78 In Bezug auf den Artikel im AStA-info wird ersichtlich, dass die Besetzung von Gärtners Büro und Teilen der Universität den öffentlichen Druck auf den Ordinarius und die Universität erhöhen sollte, um eine Amtsenthebung des vermeintlich überführten Wissenschaftlers zu erwirken. $\stackrel{79}{ }$ Weder das unbefugte Betreten der Räumlichkeiten noch die Forderung der Amtsenthebung Gärtners vor dem versammelten Senat führten letztendlich dazu, dass Professor Gärtner seine Stellung aufgeben musste.

Nicht nur in den weiteren Aktionsformen unterschied sich die Vorgehensweise der Kieler Studenten Jungjohann, Schlinke und Schmelzer von den Journalisten des linken Hamburger Blattes, sondern auch in ihrem Verhalten während der juristischen Auseinandersetzungen. Obwohl aus einem Bericht der Kieler Nachrichten hervorgeht, dass die drei Studenten „die Behauptung [gegen Gärtner], nicht aufrecht erhalten [und] es ihnen ferngelegen habe, den Kläger persönlich zu diffamieren“, nur wenige Monate später die Beharrlichkeit ihrer Aussage. Denn die drei Studenten versuchten, den Spieß umzudrehen, indem sie im März 1970 Strafanzeige wegen 
Verleumdung gegen den Kieler Ordinarius erstatteten. Als Grundlage führten sie an, dass Professor Gärtner in einer Stellungnahme erklärt habe, „den Studenten sei es allein darauf angekommen, die ,konkret'-Veröffentlichungen ,für revolutionäre Umtriebe im Universitätsleben auszuschlachten' und mit ihrer Hilfe einen ,handfesten Skandal' zu inszenieren".․․ Die Widerklage wurde vom Landgericht abgewiesen. Zum Ende des zivilrechtlichen Verfahrens gegen die AStA-Mitarbeiter wurde, wie oben beschrieben, nur der ehemalige Vorsitzende Dietmar Schlinke dazu verurteilt, einen Widerruf zu den „übernommenen Beschuldigungen gegen Gärtner“ zu veröffentlichten..$\underline{\text { a }}$ Der schriftliche Widerruf sollte bereits im Juni 1972 erscheinen. Da in den Prozessakten zur Horst Gärtner-Affäre kein solcher Widerruf zu finden ist, scheint dieser folglich nicht veröffentlicht worden zu sein. Der Kontakt des Oberlandesgerichts Schleswig zu Schlinke war aufgrund seines Aufenthalts in Kalifornien anscheinend abgebrochen, wie aus dem Bericht der Kieler Nachrichten ersichtlich wird. $\underline{\underline{83}}$ Der persönliche Einsatz der drei Beklagten ließ im Lauf des Verfahrens immer mehr nach und brach irgendwann - wie am Beispiel Schlinke zu sehen ist - gänzlich ab.

\section{Professor Gärtner und die Kieler Universität: Der Kampf um Rehabilitierung}

Die konkret-Reportage und ihre Folgen führten bei dem Kieler Ordinarius Horst Gärtner insbesondere zu einer nachhaltigen Schädigung seines wissenschaftlichen Rufs. Seine Motivation, gegen die Verleumdungen vorzugehen, beruhte in erster Linie darauf, seinen Ruf weitestgehend wiederherzustellen und eine Entschädigung für die dadurch entstandenen Beeinträchtigungen zu erhalten. Dies wird anhand seiner weiteren Vorgehensweise ersichtlich. Neben Horst Gärtner werden nachstehend ebenso der akademische Mittelbau des Hygiene-Instituts, der Senat sowie das Kultusministerium näher betrachtet, um die Vorgehensweise der gesamten Institution Universität aufzuzeigen.

Bis zur Senatssitzung am 14. Oktober 1969 hatte sich Gärtner lediglich in Form einer Erinnerungsnotiz, die er an den Kurator der Universität Kiel, den echten Ministerialrat Strathmann im Bundesverteidigungsministerium und seinen Rechtsanwalt weiterleitete, zu dem fingierten Telefongespräch und den Verleumdungen geäußert. Hierin erklärte er, dass er in dem Gespräch keinen Forschungsauftrag entgegengenommen und auch nie im Auftrag des Bundesverteidigungsministeriums gearbeitet habe. $\frac{84}{4}$ In dem konkret-Artikel „Der Giftmischer von Kiel“, der widerrechtlich die Senatssitzung vom 14. Oktober 1969 wiedergab, wurden die studentischen Vertreter Schlinke und Stabel als Ankläger Professor Gärtners stilisiert, die diesen in ein Kreuzverhör 


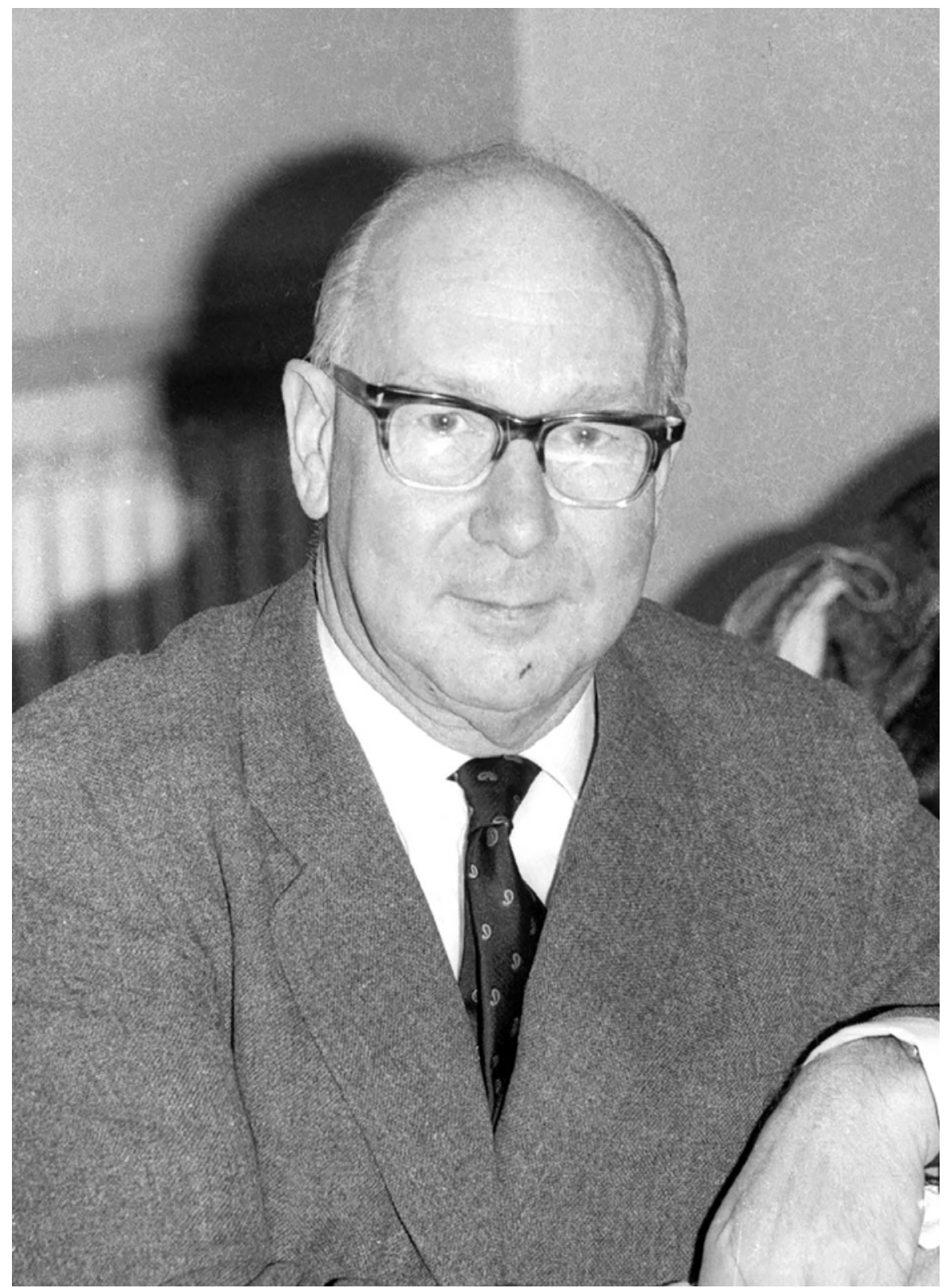

Abb. 12: Prof. Dr. Horst Gärtner, Direktor des Hygiene-Instituts der CAU (16.1.1968)

(C) 1968 Friedrich Magnussen, veröffentlicht unter der Lizenz CC-BY-SA 3.0 DE, Stadtarchiv Kiel, Sign. 42.585 
nahmen. $\frac{85}{}$ Interessant ist, dass Professor Wolfgang Naucke, Ordinarius der Rechtsund Staatswissenschaftlichen Fakultät, im gleichen Artikel besonders hervorgehoben wurde. Den abgedruckten Äußerungen des Juristen zufolge erschien es, als spreche sich dieser in gewisser Weise gegen Gärtner und für die Studierenden aus. $\underline{86}$

Obwohl Rektor Ludwig Weisbecker in dem Artikel kaum erwähnt wurde, lassen sich doch dessen Grundprinzipien aus der Stellungnahme des Rektorats in der Nordwoche vom 12. Dezember 1969 herauslesen. Dieser hatte sein gesamtes Rektoratsjahr auf Deeskalation ausgerichtet und versucht, zwischen den beiden Parteien Professoren und Studierenden - zu vermitteln, obwohl er die gewaltsamen Methoden der antiautoritären Bewegung verurteilte. $\frac{87}{7}$ Die Tatsache, dass auf der Senatssitzung im Oktober eine Untersuchungskommission gebildet wurde, die den Wahrheitsgehalt der Gerüchte um Professor Gärtner ermitteln sollte, und dass in dieser Studierende vertreten waren, zeigte, dass man gewillt war, der Forderung der Studierenden nach mehr Einsicht und Kontrolle von Forschungsprojekten entgegenzukommen. Gleichzeitig kritisierte das Rektorat aber auch, dass einerseits die Finanzierung durch das Land unzulänglich sei, weswegen auf Dritte zurückgegriffen werden müsse. Andererseits bemerkte es kritisch, dass „die Möglichkeiten der Information, die die Universität anbietet, von der Presse und der Öffentlichkeit nur unzureichend bzw. schlechter als in der Vergangenheit genutzt“ wurden. $\frac{88}{2}$ Zudem lässt sich erkennen, dass auch Professor Weisbecker eine neue Hochschulgesetzgebung für überfällig und dringend erforderlich hielt. $\stackrel{89}{ }$ Denn damit „würde auch der bloße Verdacht solcher ungeheuerlichen Vorwürfe auf Grund der korporativen Selbstkontrolle ausgeräumt werden“. $\underline{90}$

Der Vorschlag zur Bildung einer Untersuchungskommission wurde dabei dem Senat von Professor Gärtner selbst unterbreitet. Er kam seinen Anklägern somit zuvor, um die Anschuldigungen vor Ort aus der Welt räumen zu können und seinen Ruf wiederherzustellen.

Darüber hinaus ging Professor Gärtner allerdings mittels strafrechtlicher und zivilrechtlicher Verfahren gegen die konkret und die Kieler Studenten vor und verließ damit den ausgleichenden Weg. So erläuterte das Kultusministerium bereits am 16. Oktober 1969 in einer Pressemitteilung, es prüfe, „ob die Darstellungen der Zeitschrift und des studentischen Informationsblattes strafbar“ seien. 1 Nur einen Tag später und noch vor dem Erscheinen des konkret-Folgeartikels zu der Senatssitzung vom 14. Oktober 1969 kündigte das Kultusministerium an, Strafantrag wegen Verleumdung gegen die konkret zu stellen. $\frac{92}{}$ Zudem wurde aus der Pressemitteilung ersichtlich, dass bereits zivilrechtliche Schritte gegen die konkret-Journalisten sowie die Kieler AStA-Mitarbeiter eingeleitet worden waren. Das Kultusministerium begleitete die laufenden Gerichtsprozesse und trat im Strafverfahren als Nebenkläger auf. $\underline{93}$ 
Zunächst scheint es, dass Professor Gärtner als einziger der in der Reportage beschuldigten Wissenschaftler strafrechtliche Schritte eingeleitet hatte. Aus einem Artikel des General-Anzeigers aus Bonn geht allerdings hervor, dass auch Professor Klimmer, der wie Professor Gärtner in dem konkret-Artikel der Kriegsforschung bezichtigt wurde, die Verleumdungen der Bonner Studierenden - die den Ordinarius wegen des konkret-Artikels als "Multimenschenmörder“ bezeichnet hatten - strafrechtlich verfolgen wollte. ${ }^{94}$ Im Gegensatz zu Rektor Weisbeckers Versuchen, zwischen Studierenden und Ordinarien zu vermitteln, um allen Gehör zu verschaffen, wählte Gärtner einen kompromisslosen, wenn auch legalen Weg. Damit schloss er sich wie auch Professor Klimmer einem durchaus gängigen Vorgehen seitens der Professoren an, die sich gegen die 68er-Bewegung und ihre Methoden zur Wehr setzten. Mit seinen Maßnahmen gegen die Kieler Studenten näherte sich Gärtner dem bundesweiten Ordnungsrecht von 1969 an, das vom Kultusministerium des Landes Schleswig-Holstein angestrebt wurde und ein härteres Disziplinarverfahren gegen protestierende Studierende vorsah. Wie er sich tatsächlich zum Ordnungsrecht positionierte, welches sowohl vonseiten der Hochschule als auch der Studierenden abgelehnt wurde, kann an dieser Stelle nicht nachvollzogen werden. $\cdot 5$

Als letzte Akteure werden im Folgenden kurz der Akademische Mittelbau und dessen Vorgehensweise betrachtet. Denn nicht nur Professor Gärtner wurde durch die Anschuldigungen des linken Blattes diskreditiert, sondern auch sämtliche Mitarbeiter des Hygiene-Instituts.

Um ihren Vorgesetzten Professor Gärtner zu entlasten, gaben „sämtliche[!] wissenschaftliche[!] Mitarbeiter, Sekretärinnen, Haushaltssachbearbeiterinnen und leitende[!] Laborassistentinnen des Instituts“ eine eidesstattliche Erklärung ab und bestätigten, „daß entsprechende Versuche im Institut nicht durchgeführt worden“ seien. $\frac{96}{}$ Interessant ist, dass sich einige andere Assistentinnen und Assistenten der Kieler Hochschule anonym ebenfalls zu den Vorfällen und Verleumdungen äußerten. Sie stellten sich wider Erwarten nicht auf Gärtners Seite. Stattdessen taten sie ihren Unmut bezüglich der aktuellen hochschulpolitischen Verhältnisse kund. In dem Informationsblatt Der Rote Assistent wurde in dem Artikel „Zum Fall Gärtner: Kriegsforschung in der BRD“ analog zu AStA-info die „Öffentlichkeit aller Forschungsergebnisse“ verlangt. ${ }^{97}$ Diese Reaktion auf die Vorwürfe kann darauf zurückgeführt werden, dass den Assistentinnen und Assistenten aufgrund der noch bestehenden Ordinarienuniversität der Einblick in die Forschungsprojekte der Professoren beziehungsweise in die Finanzierung der selbigen verwehrt blieb. Auch sie forderten nun mehr Transparenz und Mitbestimmung an den Universitäten. Weitere kritische Äußerungen hinsichtlich der hochschulpolitischen Verhältnisse seitens des Akademischen Mittelbaus bieten die Verfahrensakten jedoch nicht. Abgesehen von der eidesstattlichen Versiche- 
rung der Angehörigen des Hygiene-Instituts lässt sich auf Grundlage der Quellen nur vermuten, dass es zu Zeugenaussagen vor dem Kieler Landgericht im zivilrechtlichen Verfahren gegen die Verfasser des AStA-info kam, die in einem Bericht der Kieler Nachrichten angedeutet werden: „Wenn der Vergleichsvorschlag nicht angenommen wird, sollen voraussichtlich Angehörige des Hygieneinstitutes als Zeugen vernommen werden. “토 Da die Kieler Studenten Schlinke, Jungjohann und Schmelzer den Vergleichsvorschlag mittels einer Strafanzeige gegen Gärtner ablehnten, wird hier davon ausgegangen, dass auch Gärtners Angestellte am Gerichtsverfahren beteiligt waren.

\begin{tabular}{|c|c|c|c|}
\hline 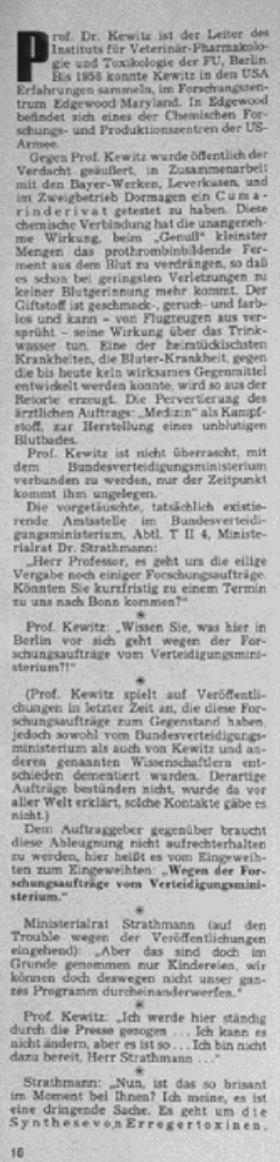 & 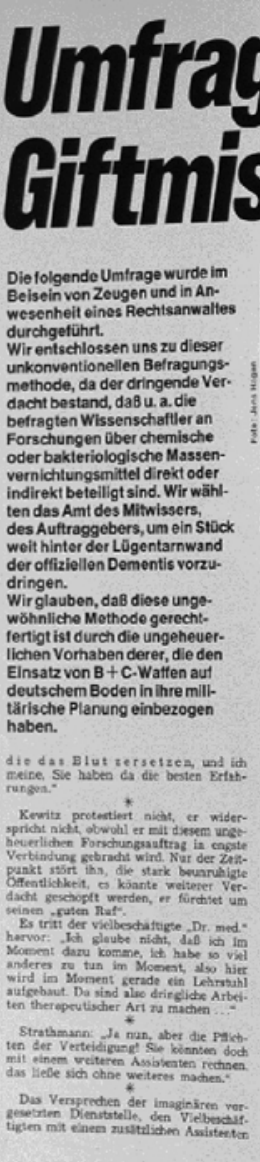 & 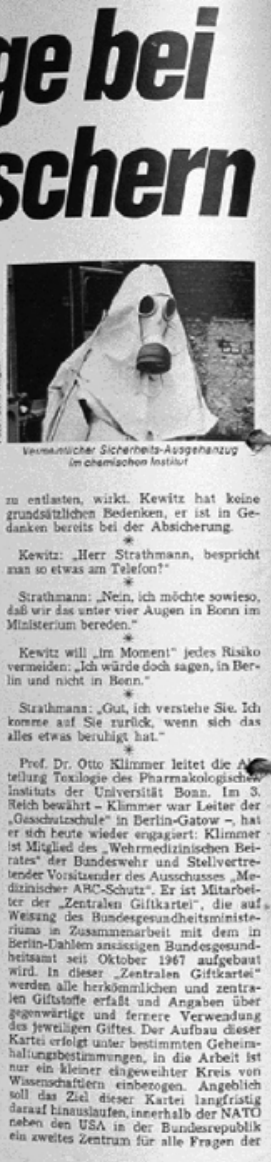 & 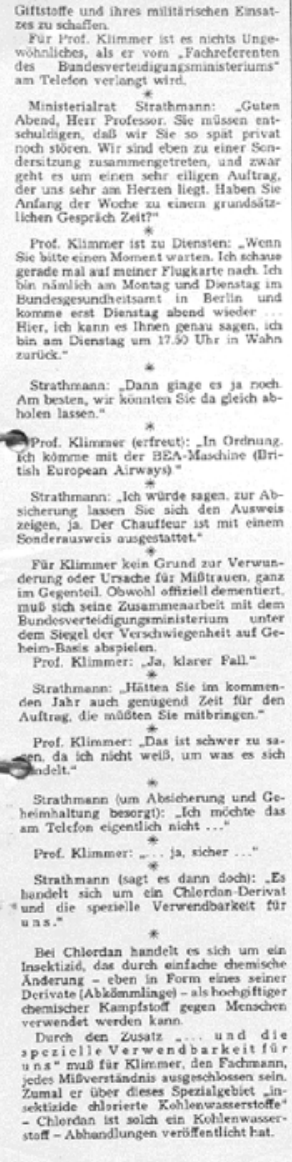 \\
\hline
\end{tabular}

Abb. 13: Artikel ,Umfrage bei Giftmischern' aus konkret Nr. 21 (2.10.1969).

Alle Rechte vorbehalten (Kvv Konkret Vertriebsgesellschaft Für Druck und Andere Medien GmbH \& Co. KG), Quelle: Landesarchiv Schleswig-Holstein, Abt. 811, Nr. 21094. 


\section{Die Horst Gärtner-Affäre: Ein Fazit}

Mit der 68er-Bewegung begann ein transnationaler Umbruch, der nicht nur in den großen Metropolen der Welt zu sehen war. Auch auf Kiel schwappte die Welle der antiautoritären Bewegung über, die sich dem Kampf gegen die Regierung, das Establishment und insbesondere ihre Professoren verschrieben hatte. Die Horst GärtnerAffäre ist nur ein Beispiel von vielen der Kieler Universität, das zeigt, welche Methoden sich die linke Protestbewegung dabei zu eigen machte. Zentrales Mittel sind hier die Massenmedien wie die Hamburger Zeitschrift konkret, um Aufmerksamkeit zu erregen. So auch mit der Reportage „Giftgas für die Bundeswehr“, die auf einen Gesichtsverlust der Bundeswehr, des Bundesverteidigungsministeriums und der Bonner Regierung abzielte. Der Kampf gegen zentrale Institutionen der BRD schloss die Diskreditierung westdeutscher Professoren wie Professor Gärtner mit ein, die angeblich an Forschungsprojekten für militärische Zwecke arbeiteten. Die haltlosen Anschuldigungen gegen den Kieler Ordinarius allein reichten aus, um den Kieler Studierenden einen Ansatzpunkt für ihre eigenen Forderungen zu bieten. Die Reportage, die sich eigentlich gegen die gesamte BRD richtete, wurde zum Anstoß für die Studierenden vor Ort, die Kieler Hochschule unter Druck zu setzen, um eine Veränderung des bestehenden Hochschulsystems mit Aussicht auf mehr Teilhabe zu bewirken.

Mit dem Beginn des Jahres 1970 brachen die extrem provokativen, aber gewaltfreien Aktionen seitens der konkret-Journalisten beziehungsweise des Redakteurs Klaus Rainer Röhl gegen Horst Gärtner ab. Die Verfahrensakten verzeichnen zumindest keine weiteren Veröffentlichungen seitens der konkret gegen den Kieler Ordinarius oder dessen Kollegen mehr. Die Studentenbewegung hatte sich parallel zu den laufenden Gerichtsverfahren zunehmend bis hin zu einem gewaltbereiten Linksextremismus radikalisiert. Im Falle der konkret gibt es diesbezüglich einen wichtigen Aspekt, der mit dem Abklingen ihres provokativen Verhaltens in Zusammenhang steht. Im Jahr 1970 zeichnete sich bereits der drei Jahre später eintretende Konkurs des linken Blattes ab, denn „[a]ls [Ulrike] Meinhof 1970 zur Terroristin wurde“, begann Klaus Rainer Röhl

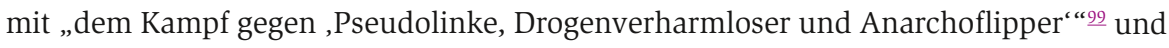
damit, sich von „seiner ehemaligen Klientel“ ${ }^{100}$ abzuwenden. Der Radikalisierungsprozess hinterließ demnach auch beim Zentralorgan der Außerparlamentarischen Opposition unweigerlich seine Spuren. $\frac{101}{}$

Nichtsdestotrotz gelang es der konkret mit ihren provokativen Publikationen, die Kieler Studierendenschaft gegen Gärtner aufzuwiegeln. Die Vorwürfe gegen Professor Gärtner und seine Kollegen in dem nachfolgenden Bericht vom 2. Oktober 1969, an B- und C-Waffen mitgearbeitet zu haben, fanden daher schnell ihren Weg an die Kieler Universität. Die Kieler Studenten handelten wiederum vor Ort. Sie nutzten dafür nicht 
nur die universitätsinternen Medien, sondern auch ihre Ämter als Studentenvertreter in der Senatssitzung, um Kritik an den mangelnden Einblicken in die akademischen Forschungsprojekte zu äußern. Ziel war es, mittels dieser Kritik eine Änderung des Hochschulsystems herbeizuführen. Sie gingen auf Konfrontationskurs mit dem Beschuldigten. Aus ihrem Aktionismus - die Besetzung von Gärtners Räumlichkeiten sowie Teilen der Universität - und den vorangegangenen, unkritisch recherchierten Publikationen lassen sich eindeutig die zentralen Beweggründe der Kieler Protestbewegung ausmachen. Sie forderten mehr Transparenz bei wissenschaftlichen Forschungsprojekten, eine objektive Kontrolle und eine Drittelparität, um den potentiellen Missbrauch von Forschung durch die Regierung oder die Bundeswehr zu verhindern. Ausschlaggebend für die Forderungen ist hier die noch ausstehende Hochschulreform. Professor Gärtner wurde im Gegensatz zu seinen Kollegen Eyer und Klimmer nicht einer nationalsozialistischen Vergangenheit bezichtigt. Dieser Umstand verdeutlicht noch einmal, dass es der Kieler Studentenschaft in diesem Fall vorrangig um eine Hochschulreform im Sinn von Demokratisierung ging.

Professor Gärtners Motivation, gerichtlich gegen die Protestbewegung vorzugehen, wurde durch die Verleumdungen in dem Bericht „Umfrage bei Giftmischern“ ausgelöst. Für den Ordinarius war die einzige Chance auf Rehabilitierung, rechtliche Schritte gegen die konkret-Journalisten und die drei Kieler Studenten einzuleiten. Mit dem Rückhalt aus dem Ministerium gelang ihm immerhin, dass die Aktionen gegen seine Person eingestellt wurden. Durch die Reportage und die darauffolgenden Berichte erfuhr der Ordinarius jedoch eine langanhaltende Schädigung seines Rufs. Jahrelang kämpfte Gärtner um Rehabilitierung. Trotz der Schwierigkeiten blieb er bis 1979 im Amt eines ordentlichen Professors an der CAU tätig und beendete seine Karriere im Jahr 1980 als Lehrbeauftragter an der Kieler Universität.

Nele Dittrich studiert die Fächer Germanistik und Geschichte im Lehramt an der Christian-Albrechts-Universität zu Kiel.

Kontakt:neledittrich@gmx.de 


\section{Anmerkungen}

Der Beitrag ist unter der Creative-Commons-Lizenz Namensnennung 4.0 international veröffentlicht. Den Vertragstext finden Sie unter: https://creativecommons.org/licenses/by/4.0/deed.de. Bitte beachten Sie, dass einzelne, entsprechend gekennzeichnete Teile des Werks von der genannten Lizenz ausgenommen sein bzw. anderen urheberrechtlichen Bedingungen unterliegen können.

1 Der vorliegende Artikel begründet sich auf der gleichnamigen Bachelorarbeit, die 2018 im Rahmen des Kieler Gelehrtenverzeichnisses entstand.

$\underline{2}$ Oliver Auge (Hg.), Christian-Albrechts-Universität zu Kiel. 350 Jahre Wirken in Stadt, Land und Welt, Kiel/Hamburg 2015. Vgl. hier die Beiträge von Rainer S. Elkar, Beteiligung und Verantwortung. Ausschnitte einer studentischen Geschichte zu Kiel, S. 561-610; Wilfried Müller, Die Kieler Studierendenbewegung. Eine persönliche Chronologie, S. 611-621; Stefan Bichow, „Verfolgung und Ermordung der Universitätswürde 1968". Die Studentenproteste an der Christian-Albrechts-Universität, S. 622-636.

$\underline{3}$ Mit der Jahrtausendwende rückte die 68er-Bewegung zunehmend in den Fokus der deutschen Geschichtsforschung. Zu nennen sind beispielsweise Wolfgang Kraushaar, 1968 als Mythos, Chiffre und Zäsur, Hamburg 2000; Norbert Frei, 1968. Jugendrevolte und globaler Protest, München 2008; Kathrin Fahlenbrach, Protestinszenierungen. Die Studentenbewegung im Spannungsfeld von Kultur-Revolution und Medien-Evolution, in: 1968. Handbuch zur Kultur- und Mediengeschichte der Studentenbewegung, hg. von Martin Klimke und Joachim Scharloth, Stuttgart 2007, S. 11-21. Seit 2009 wurden zunehmend Beiträge zur Kieler Studentenbewegung publiziert - unter anderem von Christoph Cornelißen, „Kiel 68" - Sozialprotest und kultureller Aufbruch, in: Mitteilungen der Gesellschaft für Schleswig-Holsteinische Geschichte 85 (2009), H. 1, S. 1-5; Rainer S. Elkar, Studieren in Kiel. Eine historisch-politische Zeitreise von den Anfängen bis zur Gegenwart, Husum 2015 (Sonderveröffentlichung der Gesellschaft für Kieler Stadtgeschichte, Bd. 77); Stefan Bichow, Die Universität Kiel in den 1960er Jahren. Ordnungen einer akademischen Institution in der Krise, (Kieler Werkstücke H/3), Frankfurt am Main u. a. 2013; Swantje Piotrowski, Kieler Professoren, ihre Söhne und der linke Terrorismus. Zum Umgang mit akademischem Traditionsbewusstsein und politischer Radikalisierung an der CAU, (in diesem Band). Als Quellen wurden drei Akten aus dem Landesarchiv Schleswig-Holstein (LASH) herangezogen. Es handelt sich hierbei um die Personalakte Gärtners, LASH, Abt. 47, Nr. 2140, sowie zwei Verfahrensakten des Kultusministeriums, anhand derer der Verlauf der Affäre rekonstruiert werden kann. Vgl. zu Letzterem LASH, Abt. 811, Nr. 21094; LASH, Abt. 811, Nr. 21097. Weitere Quellen konnten aus datenschutzrechtlichen Gründen nicht eingesehen werden.

$\underline{4}$ Vgl. „Der Giftmischer von Kiel. Folgen einer Reportage”, in: konkret 23 (1969), S. 39-41.

$\underline{5}$ Pressemitteilung des Kultusministers des Landes Schleswig-Holstein, 16.10.1969, LASH, Abt. 47, Nr. 21094.

$\underline{6}$ Vgl. ebd.

$\overline{7}$ Vgl. „Prof. Gärtner gegen ,konkret'. Der Rechtsstreit ist noch nicht geklärt - Weiterer ,Schriftverkehr'”, in: Kieler Nachrichten (06.12.1969). LASH, Abt. 47, Nr. 2140.

8 Vgl. „Röhl muß widerrufen. Professor Gärtner auch in Zivilprozessen erfolgreich“, in: Kieler Nachrichten (18.11.1972). LASH, Abt. 811, Nr. 21094.

$\underline{9}$ „Wegen übler Nachrede verurteilt", in: Kieler Nachrichten (11.11.1972). LASH, Abt. 811, Nr. 21094.

10 Vgl. Frederik Obermaier, Sex, Kommerz und Revolution. Vom Aufstieg und Untergang der Zeitschrift konkret (1957-1973), Marburg 2011, S. 78-82. Vor ihrer Neugründung war die konkret eine Studentenzeitschrift, die bis 1957 noch StudentenKurier hieß.

11 Bei Manfred Gfellschild handelt es sich um ein Pseudonym. Während der laufenden Verfahren konnte nicht festgestellt werden, wer sich hinter diesem Namen verbarg. Erst 1998 gab Wallraff den wahren Namen seines Mitautors preis. Es handelt sich um den Journalisten Jörg Heimbrecht. Vgl. hierzu Markus Krischer und Rainer Schmitz „Affäre: Anruf von Wallraff”, in: Focus Magazin 26 (1998), https://www.focus.de/auto/neuheiten/affaere-anruf-von-wallraff aid 170660.html (08.03.2020, 11:56 Uhr).

12 Jochen Staadt, "Giftgas für die Bundeswehr", in: Frankfurter Allgemeine Zeitung (04.09.2003), https://www.faz.net/aktuell/ politik/guenter-wallraff-giftgas-fuer-die-bundeswehr-1115215.html (23.02.2020, 10:41 Uhr). Bakteriologische Waffen fallen unter die Regelungen zu biologischen Waffen, vgl. hierzu Genfer Protokoll - Protokoll über das Verbot der Verwendung von erstickenden, giftigen oder ähnlichen Gasen sowie bakteriologischen Mitteln im Kriege, Genf 17.6.1925.

13 "Giftgas für die Bundeswehr", in: konkret 21 (1969), S. 12-15.

14 Wolfgang Kraushaar, Die Furcht vor einem „neuen 33". Protest gegen die Notstandsgesetzgebung, in: Streit um den Staat. Intellektuelle Debatten in der Bundesrepublik 1960-1980, hg. von Dominik Geppert und Jens Hacke, Göttingen 2008 S. 135-151, hier S. 135 und 138; vgl. ders., Achtundsechzig. Eine Bilanz, Berlin 2008, S. 165f.; vgl. Obermaier, Sex, Kommerz und Revolution (wie Anm. 10), S. 91f. Damit waren zwei Arten von Notstand gemeint: der innere sowie der äußere Notstand. Sollte es zu einem Krieg kommen, würde dies als äußerer Notstand gelten. Innerer Notstand bedeutete, dass im Falle von beispielsweise Naturkatastrophen oder einem Generalstreik „der Bundestag bzw. [...] auch der Bundespräsident bei Gegenzeichnung durch den Bundeskanzler berechtigt werden [sollte], den Ausnahmezustand [...] zu verhängen". Vgl. hierzu Kraushaar, "neuen 33", S. 135. Letzteres wurde nicht nur von der Widerstandsbewegung außerhalb der Regierung als problematisch angesehen, sondern auch durch Politiker aus den Reihen der SPD kritisiert. Ein Machtmissbrauch durch derlei besondere Gesetzgebungsbefugnisse konnte nicht ausgeschlossen werden, da die Notstandsgesetzgebung an die Weimarer Verfassung erinnerte.

15 "Prof. Gärtner ist kein Giftmischer", in: konkret 24 (1969), LASH, Abt. 811, Nr. 21094.

16 "Giftgas für die Bundeswehr" (wie Anm. 13), S. 12.

$\underline{17}$ Vgl. Trägerverein „Vereinigung der Freundinnen und Freunde der Neuen Wege" (Hg.): Neue Wege. Beiträge zu Religion und Sozialismus, 1969, S. 43-50, www.e-periodica.ch/cntmng?pid=new-001:1969:63::439 (15.2.2020, 19:15 Uhr). 
18 Vgl. Matthias N. Lorenz, Art. „IV. A5 Rücktritt Heinrich Lübke“, in: Lexikon der „Vergangenheitsbewältigung" in Deutschland. Debatten- und Diskursgeschichte des Nationalsozialismus nach 1945, hg. von Torben Fischer und dems., Bielefeld 2007, S. 187-189; vgl. Wolfgang Kraushaar, Denkmodelle der 68er-Bewegung, in: Aus Politik und Zeitgeschichte 51 (2001), S. 14-27, hier S. 19f.; vgl. Frei, 1968 (wie Anm. 3), S. 82-84.

19 Vgl. hierzu Obermaier, Sex, Kommerz und Revolution (wie Anm. 10), S. 47-76; vgl. auch Frei (wie Anm. 3), 1968 , S. 84.

20 Obermaier, Sex, Kommerz und Revolution (wie Anm. 10), S. 76.

21 Vgl. Michael Ludwig Müller, Die DDR war immer dabei. SED, Stasi \& Co. und ihr Einfluss auf die Bundesrepublik, 2014 Reinbek/München, S. 209-211; Obermaier, Sex, Kommerz und Revolution (wie Anm. 10), S. 109f.; Markus Krischer und Rainer Schmitz, "Zeitgeschichte. Auskunft über ,Wagner"', in: Focus Magazin 22 (1998), https://www.focus.de/politik/ deutschland/zeitgeschichte-auskunft-ueber-wagner aid 169065.html (08.03.2020, 19:52 Uhr); Jürgen Dahlkamp und Georg Mascolo, „Stasi-Akten. Die verlorene Ehre des Günter W.”, Der Spiegel 36 (2003), https://www.spiegel.de/spiegel/ print/d-28471045.html (08.03.2020, 19:49 Uhr).

22 Vgl. Neele Kerkmann, Art. „IV. A3 Kiesinger-Ohrfeige”, in: Lexikon der „Vergangenheitsbewältigung" in Deutschland. Debatten- und Diskursgeschichte des Nationalsozialismus nach 1945, hg. von Torben Fischer und Matthias N. Lorenz, Bielefeld 2007, S. 185f.; Obermaier, Sex, Kommerz und Revolution (wie Anm. 10), S. 88; vgl. hierzu Wolfgang Kraushaar, 1968 und Massenmedien, in: Archiv für Sozialgeschichte 41 (2001), S. 317-347, hier S. 319 und 332.

$\underline{23}$ "Prof. Gärtner ist kein Giftmischer" (wie Anm. 15).

$\underline{24}$ Seit März 1965 widmete sich die Hamburger Zeitschrift konkret den Gräueltaten des Vietnamkriegs. Hierzu Obermaier, Sex, Kommerz und Revolution, S. 94. Die Eskalation des Vietnamkrieges wurde zum Antrieb für weltweite Protestbewegungen, denen sich die westdeutschen 68er zugehörig fühlten. Ein genereller Antiimperialismus stellte sich unter den Protestlern ein. Philipp Gassert, Antiamerikanismus und Antiimperialismus um 1968. Proteste gegen die US-Außenpolitik, in: 1968 und die 68er. Ereignisse, Wirkungen und Kontroversen in der Bundesrepublik, hg. von Gerrit Dworok und Christoph Weißmann, Wien u.a. 2013, S. 153-170, hier S. 163. Die westdeutschen 68er projizierten ihren eigenen Kampf gegen das deutsche Establishment auf die Revolutionen in der ganzen Welt. Vgl. Kraushaar, Denkmodelle (wie Anm. 18), S. 24. Zuvor war es die Revolution in Lateinamerika, die der westdeutschen Studentenbewegung als Vorbild diente.

25 Ebd.

26 Vgl. "Umfrage bei Giftmischern”, in: konkret 21 (1969), S. 16-19; vgl. Obermaier, Sex, Kommerz und Revolution (wie Anm. 10), S. 109; vgl. Müller, Die DDR war immer dabei (wie Anm. 21), S. 211-216.

$\underline{27}$ Vgl. „Umfrage bei Giftmischern” (wie Anm. 26)

28 Vgl. Kraushaar, Mythos (wie Anm. 3), S. $37 f$.

$\underline{29}$ "Umfrage bei Giftmischern" (wie Anm. 26), S. 18.

30 Hans-Ulrich Thamer, Die NS-Vergangenheit im politischen Diskurs der 68er-Bewegung, in: Westfälische Studien 48 (1998) S. 39-53, hier S. 51. Auch die Kieler Studentenbewegung protestierte gegen mit einer NS-Vergangenheit belastete Professoren. Ein Beispiel hierfür ist die mehrere Tage andauernde Besetzung des Seminars für Wissenschaft und Geschichte der Politik im Universitätshochhaus im Juni 1969. Ziel dieser Aktion war es, „einschlägiges Material” gegen den Direktor des Seminars Michael Freund zu finden, der als "nationalsozialistisch belastet" galt. Vgl. Elkar, Beteiligung und Verantwortung (wie Anm. 2), S. 602f.

31 Thamer, NS-Vergangenheit (wie Anm. 30), S. 42

32 Vgl. Ulrich Herbert, Generationenfolge in der deutschen Geschichte des 20. Jahrhunderts, in: Generationalität und Lebensgeschichte im 20. Jahrhundert, hg. von Jürgen Reulecke, München 2003 (Schriften des Historischen Kollegs, Bd. 58), S. 95-114, hier S. 113.

$\underline{33}$ Frei, 1968 (wie Anm. 3), S. 82-84.

34 Vgl. Obermaier, Sex, Kommerz und Revolution (wie Anm. 10), S. 104f. Als Beispiele führt Obermaier zum einen die "Aktion Freie Pille" und zum anderen die Aktion zur Wehrdienstverweigerung im August 1968 an. Hieran wird ersichtlich, dass die Bundeswehr ein ständiges Thema auf den Seiten der konkret gewesen zu sein scheint.

35 Vgl. Obermaier, Sex, Kommerz und Revolution (wie Anm. 10), S. 109f.; Müller, Die DDR war immer dabei (wie Anm. 21), S. 209-216; Krischer/Schmitz, Zeitgeschichte (wie Anm. 21); Dahlkamp/Mascolo, Stasi-Akten (wie Anm. 21).

$\underline{36}$ "Der Giftmischer von Kiel. Folgen einer Reportage" (wie Anm. 4), S. 39-41.

$37 \mathrm{Ebd}$

$\underline{38}$ Ebd. Wer das Tonbandgerät - ob Ordinarius oder Student - in die Senatssitzung geschmuggelt und die Aufnahmen an die konkret weitergeleitet hat, konnte sowohl vom Amtsgericht Hamburg als auch vom Landgericht Kiel nicht festgestellt werden

39 „"Produziert Kieler Universität chemische Kampfmittel?' Stellungnahme des Rektorats der Universität Kiel“, 12. Dezember 1969 Nordwoche, LASH, Abt. 47, Nr. 2140. Bei der Kieler Nordwoche handelt es sich um eine sozialdemokratische Wochenzeitung, die von 1969-1971 herausgegeben wurde. Vgl. SPD Geschichtswerk, https://www.spd-geschichtswerkstatt.de/ wiki/Nordwoche (08.03.2020, 21:02 Uhr).

40 Vgl. „Röhl muß widerrufen. Professor Gärtner auch in Zivilprozessen erfolgreich“ (wie Anm. 8).

41 "Der Giftmischer von Kiel. Folgen einer Reportage" (wie Anm. 4), S. 39-41.

$\underline{42}$ Vgl. „PProduziert Kieler Universität chemische Kampfmittel?' Stellungnahme des Rektorats der Universität Kiel“ (wie Anm. 39).

43 Vgl. ebd.

44 Ebd.

$\underline{45}$ Ebd.

46 "Giftgas für die Bundeswehr" (wie Anm. 13), S. 12-15. 
47 Vgl. Elkar, Studieren in Kiel (wie Anm. 3), S. 143.

48 Vgl. Piotrowski, Kieler Professoren (wie Anm. 3). Die Professorensöhne Thomas Weisbecker und Georg von Rauch junior zogen 1969 nach Berlin und schlossen sich dort linksradikalen Gruppierungen an. Von Rauch starb 1971 bei einem Schusswechsel mit der Polizei, während Weisbecker 1972 bei einer Fahndungsaktion von einem Polizisten erschossen wurde.

49 Vgl. „Strafanzeige gegen Prof. Gärtner - ,Unwahre Behauptungen und schwerwiegende Beschuldigungen' gegen drei Studenten / Verhandlung am 26. März in Kiel", 19. März 1970 Flensburger Tageblatt, LASH Abt. 811, Nr. 21097.

50 Vgl. Obermaier, Sex, Kommerz und Revolution (wie Anm. 10), S. 109f; vgl. Müller, Die DDR war immer dabei (wie Anm. 21), S. 211-216; Krischer/Schmitz, Zeitgeschichte (wie Anm. 21).

$\underline{51}$ "Kriegsforschung in Kiel oder Gärtner muß Gärtner werden”, in: Asta-info 57 (1969), LASH, Abt. 47, Nr. 2140.

$\underline{52}$ Ebd.

$\underline{53}$ Vgl. Bichow, Die Universität Kiel (wie Anm. 3), S. 77.

54 Vgl. Elkar, Studieren in Kiel (wie Anm. 3), S. 152; vgl. ders., Beteiligung und Verantwortung (wie Anm. 2), S. 599; vgl. Bichow, Verfolgung und Ermordung (wie Anm. 2), S. $622 f$.

$\underline{55}$ Vgl. Bichow, Verfolgung und Ermordung (wie Anm. 2), S. $622 f$.

$\underline{56}$ Vgl. ebd., S. 622 und S. 626.

57 Vgl. Philipp B. Bocks, Mehr Demokratie gewagt? Das Hochschulrahmengesetz und die sozial-liberale Reformpolitik 1969-1976, Bonn 2012 (Politik- und Gesellschaftsgeschichte, Bd. 94), S. 47.

$\underline{58}$ Vgl. Bichow, Die Universität Kiel (wie Anm. 3), S. 103

$\underline{59}$ Vgl. ebd., S. 70 und S. $158 \mathrm{f}$.

$60 \mathrm{Vgl}$. ebd. Die akademische Selbstverwaltung der Hochschule gliederte sich in zwei verschiedene Verwaltungsbereiche beziehungsweise in die zentralen Verwaltungsorgane. Zum einen unterlag die Universität der Leitung des Rektors, der eine Amtsperiode von ein bis zwei Jahren innehatte, sowie dem Senat, der über die Grundsatzentscheidungen befugte. Zum anderen zeichnete sie sich "durch eine organisatorische, finanzielle und personelle Verwaltung unter Leitung eines Kanzlers bzw. Kurators (bei deutlicher Kontrolle des Staates) aus". Vgl. Bocks, Mehr Demokratie gewagt? (wie Anm. 57), S. 39. Den Professoren oblag unter anderem der Bereich der Forschungsverwaltung.

61 Vgl. ebd., S. 37.

$\underline{62}$ Vgl. Cornelißen, "Kiel 68" (wie Anm. 3), S. 1.

63 Vgl. ders., Von Aufbruchsstimmung zum Krisendiskurs der 1970er Jahre. Die Christian-Albrechts-Universität im Spiegel ihrer Rektoratsreden, in: Wissenschaft im Aufbruch. Beiträge zur Wiederbegründung der Kieler Universität nach 1945, hrsg. von dems., Essen 2014 (Mitteilungen der Gesellschaft für Kieler Stadtgeschichte, Bd. 88), S. 182-209, hier S. 192-195; vgl. Bichow, Verfolgung und Ermordung (wie Anm. 2), S. 632. Die Anzahl der Lehrstühle hatte sich bis zum Jahre 1966 bereits verdoppelt.

64 Vgl. hierzu und zum Folgenden Elkar, Studieren in Kiel (wie Anm. 3), S. 147f.; vgl. auch ders., Beteiligung und Verantwortung (wie Anm. 2), S. 597.

$\underline{65}$ Vgl. Bichow, Die Universität Kiel (wie Anm. 3), 103

66 Vgl. Vgl. ders., Verfolgung und Ermordung (wie Anm. 2), S. 629f.; Elkar, Beteiligung und Verantwortung (wie Anm. 2), S. 601f.

$67 \mathrm{Vgl}$. Elkar, Studieren in Kiel (wie Anm. 3), S. 155.

68 Vgl. Bichow, Verfolgung und Ermordung (wie Anm. 2), S. 629f.; vgl. ders., Die Universität Kiel (wie Anm. 3), S. 98-100.

69 Ebd.

$\underline{70}$ Vgl. „Der Giftmischer von Kiel. Folgen einer Reportage” (wie Anm. 4), S. 39-41.

71 Ebd., S. 39.

$\overline{72}$ Ebd., S. 39-41.

$\underline{73} \mathrm{Vgl}$. „Untersuchungskommission gewählt. Vorwürfe gegen Prof. Gärtner werden geprüft - Strafantrag gestellt", 31. Oktober 1969 Kieler Nachrichten, LASH, Abt. 47, Nr. 2140

$\underline{74}$ "'Produziert Kieler Universität chemische Kampfmittel?' Stellungnahme des Rektorats der Universität Kiel" (wie Anm. 39).

$\underline{75} \mathrm{Vgl}$. „Strafanzeige gegen Prof. Gärtner - ,Unwahre Behauptungen und schwerwiegende Beschuldigungen' gegen drei Studenten / Verhandlung am 26. März in Kiel" (wie Anm. 49).

76 Vgl. Obermaier, Sex, Kommerz und Revolution (wie Anm. 10), S. 109f; vgl. Müller, Die DDR war immer dabei (wie Anm. 21), S. 211-216; Krischer/Schmitz, Zeitgeschichte (wie Anm. 21).

77 Kraushaar, Mythos (wie Anm. 3), S. 67.

78 Ebd.

$\underline{79}$ Vgl. Obermaier, Sex, Kommerz und Revolution (wie Anm. 10), S. 110.

80 "Vergleichsvorschlag im Verfahren Prof. Gärtner kontra Studenten. Beklagte sollen ihre Behauptung zurücknehmen", 13. Dezember 1969 Kieler Nachrichten, LASH, Abt. 47, Nr. 2140.

81 Vgl. „Strafanzeige gegen Prof. Gärtner - ,Unwahre Behauptungen und schwerwiegende Beschuldigungen' gegen drei Studenten/Verhandlung am 26. März in Kiel" (wie Anm. 49).

$\underline{82}$ "Röhl muß widerrufen. Professor Gärtner auch in Zivilprozessen erfolgreich" (wie Anm. 8).

$83 \mathrm{Vgl}$. ebd.

$\underline{84}$ Vgl. Erinnerungsnotiz Professor Gärtners, 7. Oktober 1969, LASH, Abt. 47, Nr. 2140.

85 Vgl. „Der Giftmischer von Kiel. Folgen einer Reportage”, (wie Anm. 4), S. 39-41. Wie sich die restlichen Senatsmitglieder ihrem Kollegen gegenüber verhielten, kann an dieser Stelle nicht weiter beurteilt beziehungsweise betrachtet werden, da es sich bei dem Protokoll der Senatssitzung um ein vertrauliches, der Öffentlichkeit nicht zugängliches Dokument handelt.

86 Vgl. ebd. 
87 Vgl. Piotrowski, Kieler Professoren (wie Anm. 3), S. 3.

88 „'Produziert Kieler Universität chemische Kampfstoffe?' Stellungnahme des Rektorats der Universität Kiel“ (wie Anm. 39)

89 Vgl. ebd.

90 Ebd.

91 Pressemitteilung des Kultusministers des Landes Schleswig-Holstein, 16. Oktober 1969 (wie Anm. 5).

92 Vgl. „Kultusministerium: Strafantrag wegen Verleumdung”, 17. Oktober 1969 Kieler Nachrichten, LASH, Abt. 47, Nr. 2140.

93 Vgl. die Pressemitteilung des Kultusministers des Landes Schleswig-Holstein, 16. Oktober 1969 (wie Anm. 5).

94 Vgl. „Prof. Klimmer will Verleumdungen strafrechtlich verfolgen lassen“, General Anzeiger (11.11.1969), LASH, Abt. 47, Nr. 2140. Ob Professor Klimmer tatsächlich juristische Schritte eingeleitet hat, wird in den Verfahrensakten nicht weiter ausgeführt. Interessant ist allerdings, dass auch im Falle Klimmer vom selbigen der Antrag gestellt wurde, „einen disziplinarischen Untersuchungsausschuß zur Klärung der Fragen zu bilden“. Im Gegensatz zur Lage in Kiel kam es auch tatsächlich zu mehreren Besichtigungen von Professor Klimmers Abteilung durch Untersuchungsausschuss und AStA.

$95 \mathrm{Vgl}$. Bichow, Verfolgung und Ermordung (wie Anm. 2), S. 627-632. Die Verhandlungen über das geforderte Ordnungsrecht zogen sich noch in die Länge. Nachdem selbiges als verfassungsrechtlich bedenklich eingestuft wurde, drängten die Regierung und das Kultusministerium darauf, das Recht zu erlangen, mittels Polizeieinsatz und anderer Maßnahmen gegen Störungen seitens der Studierenden vorzugehen. Die Autonomie der Kieler Hochschule wurde durch ein solches Bestreben stark gefährdet. Die Studierenden selbst lieferten durch ihre Protestaktionen, denen die Hochschule sich immer wieder ausgesetzt sah, den staatlichen Behörden nur weitere Argumente, diese Beschneidung der Autonomie voranzutreiben.

96 Pressemitteilung des Kultusministers des Landes Schleswig-Holstein, 16. Oktober 1969 (wie Anm. 5).

97 „Zum Fall Gärtner: Kriegsforschung in der BRD” in Der Rote Assistent (o. D.), LASH, Abt. 811, Nr. 21094.

98 "Vergleichsvorschlag im Verfahren Prof. Gärtner kontra Studenten. Beklagte sollen ihre Behauptung zurücknehmen" (wie Anm. 80).

99 Obermaier, Sex, Kommerz und Revolution (wie Anm. 10), S. 140f. Bevor Ulrike Meinhof sich dem linken Terror und der Roten Armee Fraktion verschrieb, arbeitete die 1934 in Oldenburg geborene Journalistin für die Hamburger Zeitschrift konkret. Trotz des Wegfalls der finanziellen Unterstützung durch die KPD blieb Meinhof weiterhin Mitglied der in West-Deutschland verbotenen Partei. Ende 1961 heirateten Röhl und Meinhof. Bereits im Februar 1968 ließ sich Meinhof von ihrem Ehemann scheiden. Vgl. der., S. 110-113; „Ulrike Meinhof”, https://de.wikipedia.org/wiki/Ulrike Meinhof $(08.03 .2020,21: 45)$.

100 Ebd., S. $140 f$.

101 Vgl. ebd., S. 88 